\title{
Ostracods (Crustacea) associated with microbialites across the Permian-Triassic boundary in Dajiang (Guizhou Province, South China)
}

\author{
Marie-Béatrice FOREL \\ State Key Laboratory of Geological Process and Mineral Resources, China University of Geosciences, \\ No. 388, Lumo Road, Wuhan 430074, People's Republic of China. \\ E-mail: mbforel@yahoo.fr
}

\begin{abstract}
Upper Permian (Changhsingian) - Lower Triassic (Griesbachian) interval of the Dajiang section, Guizhou Province, South China. 112 species belonging to 27 genera are recognized. Five new species are described: Acratia candyae sp. nov, Bairdia adelineae sp. nov., Bairdia? huberti sp. nov., Bairdia jeromei sp. nov., Orthobairdia jeanlouisi sp. nov. The unexpected survival faunas associated with microbial formations in the aftermath of the end-Permian extinction are documented for the first time. Ostracod biodiversity variations and palaeo-environmental modifications associated with microbial growth through the Permian-Triassic boundary (PTB) are discussed.
\end{abstract}

Keywords. Ostracods, Permian-Triassic, Microbialites, Dajiang, South China.

Forel M.B. 2012. Ostracods (Crustacea) associated with microbialites across the Permian-Triassic boundary in Dajiang (Guizhou Province, South China). European Journal of Taxonomy 18: 1-34. http://dx.doi.org/10.5852/ ejt.2012.19

\section{Introduction}

The end-Permian extinction, about $252 \mathrm{My}$, decimated 80 to $96 \%$ of species and $52 \%$ of families in the marine realm (e.g. Sepkoski 1984; Erwin 1993; Benton \& Twitchett 2003). Several potential geological triggers have been identified: bolide impact, oceanic anoxia and euxinia and flood basalt volcanism. It is now accepted that ocean anoxia was widespread during the latest Permian (Changhsingian), its prevalence increased near the time of the main extinction and continued during the Early Triassic. Anoxic and sulfidic waters commonly also extended into shallow-marine environments. The eruption of the Siberian Traps is now considered as the primary trigger of extinction. Global warming, ocean acidification, and possible destruction of atmospheric ozone were engendered by the release of volcanic $\mathrm{CO}_{2}$ and volatilized sedimentary organic carbon and evaporite minerals. Enhanced weathering and nutrient runoff increased pre-existing ocean anoxia (see Payne \& Clapham 2012 and references therein for details). The extinction marks a major transition in marine ecosystems: the Upper Permian benthic shelly communities from shallow marine settings were replaced by widespread microbial communities. They are abundant in low-latitude shallow-marine carbonate shelves in central Tethyan continents where they occupied similar environments to Upper Permian reefs but extended into deeper waters 


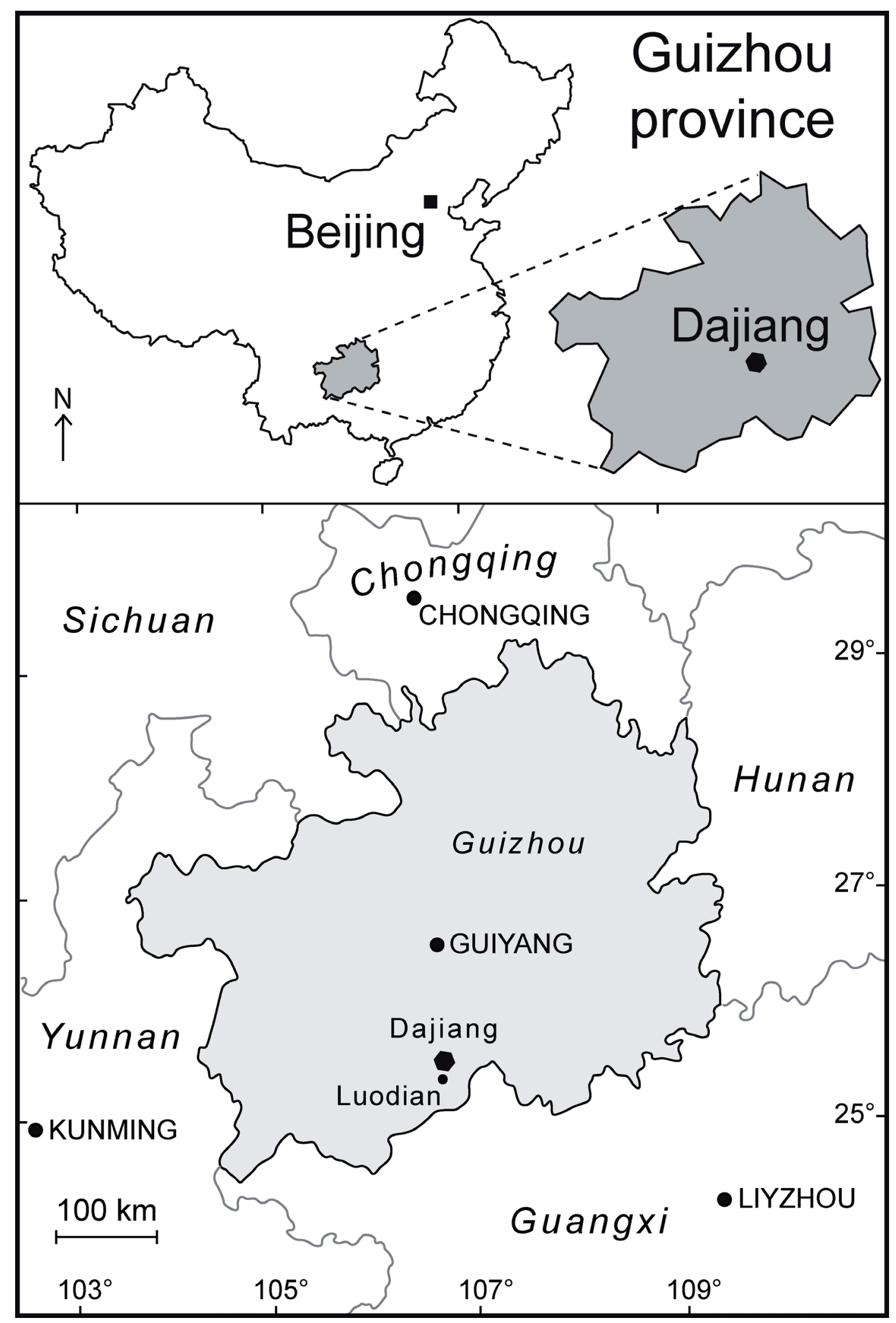

Fig. 1. Location of the Dajiang section in the southern Guizhou Province, South China. 
(Kershaw et al. 2007, 2012). Current evidence regarding oxygen levels associated with microbialites growth is conflicting : $(i)$ evidence of low-oxygen conditions (Bond \& Wignall 2010; Liao et al. 2010; Chen et al. 2011), (ii) abundant benthic shelly faunas dominated by ostracods, and occasionally some rare micro-gastropods, microbrachiopods, foraminifers and conodonts (e.g. Crasquin-Soleau \& Kershaw 2005; Forel et al. 2009; Song et al. 2009; Yang et al. 2011; Forel et al. in press). A two-steps oxygenation of the surrounding waters by cyanobacterial activity will be proposed as a possible mechanism for this unexpected survival (Forel in press).

74 to $100 \%$ of ostracods became extinct through the Permian-Triassic boundary (PTB) and very little is known about their presence and characteristics in the interval following the extinction event. In the lower Griesbachian neritic environments, they are only found in association with microbialites. The faunas described in the present article are of primary importance because they exemplify a survival phenomenon in a refuge of microbial origin documented from several localities worldwide (Forel in press; Forel et al. in press). This paper describes the upper Changhsingian and lower Griesbachian ostracod faunas of the Dajiang section (Wujiaping and Daye Formations respectively), Guizhou Province, South China. 112 species are recorded, including 5 new species, belonging to 27 genera.

\section{Material and Methods}

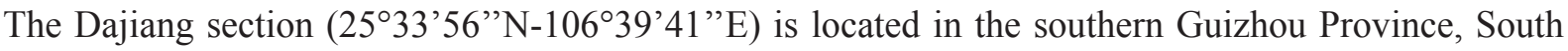
China, a few kilometres north of the city of Luodian (Fig. 1). Only a brief description of the lithological succession in Dajiang is given here: the reader is referred to Forel et al. (2009) and references therein for further details. At the base of the section are about 7 metres of open shallow marine thick, massivebedded skeletal lime-packstone of the Wujiaping Formation (Changhsingian age). They are followed by about 17 metres of microbialite carbonate layers of the Daye Formation. The conodont Hindeodus parvus (Kozur \& Pjatakova, 1976) occurs throughout the lowermost metre-thick microbialite carbonate, above the major facies change that marks the greatest loss in Permian fossils (the first occurrence of H. parvus is the index of the base of the Triassic: Yin et al. 1996; Lehrmann 1999; Lehrmann et al. 2003). The PTB 'event horizon' corresponds to the contact between the Wujiaping Formation and the microbialite (Lehrmann et al. 2003).

Samples spanning the Permian-Triassic event horizon were collected for ostracod analysis and labelled as 05PAJXx (Fig. 2). Ostracods are determined on the basis of external and internal characters of the carapace and have to be released from the enclosing matrix. Extraction by means of acid is precluded because their carapaces are made of calcium carbonate and enclosed in calcareous rocks. Samples were processed by hot acetolysis technique to disaggregate the dehydrated hard limestones and release the ostracod shells (Lethiers \& Crasquin-Soleau 1988; Crasquin-Soleau et al. 2005). Twenty-six samples were collected and analysed, only one was barren (05PAJ34; Fig. 2). The faunas in Dajiang show high intraspecific variability that hindered the clear differentiation between species in many cases. Due to the poor preservation and availability of specimens, many species are left in open nomenclature and are not listed in the systematic part (e.g. Bairdia sp. 1 to 36), although all the material of this important interval is figured. All specimens are stored in the Pierre et Marie Curie University Collections (Paris, France; collection numbers P6M2917-3150).

All the species belonging to the Bairdioidea (Sars, 1887) superfamily are shown in Figs 3 (genera Acratia Delo, 1930 and Bairdia McCoy, 1844), 4, 7-9 (genus Bairdia), 10 (genera Bairdia and Bairdiacypris Bradfield, 1935), 11 (genera Bairdiacypris, Bythocypris Brady, 1880, Fabalicypris Brady, 1880, Liuzhinia Zheng, 1976 and Orthobairdia Sohn, 1960) and 13 (genera Petasobairdia Chen, 1982, Spinocypris Kozur, 1971, Kempfina Crasquin, 2010, Silenites Coryell \& Booth, 1933 and Microcheilinella Geis, 1933). Species belonging to the Cypridoidea Baird, 1845 are gathered in Figs 13 and 14 (genus Paracypris Sars, 1866). Cytheroidea Baird, 1850 are shown in Figs 14 (genera Monoceratina 


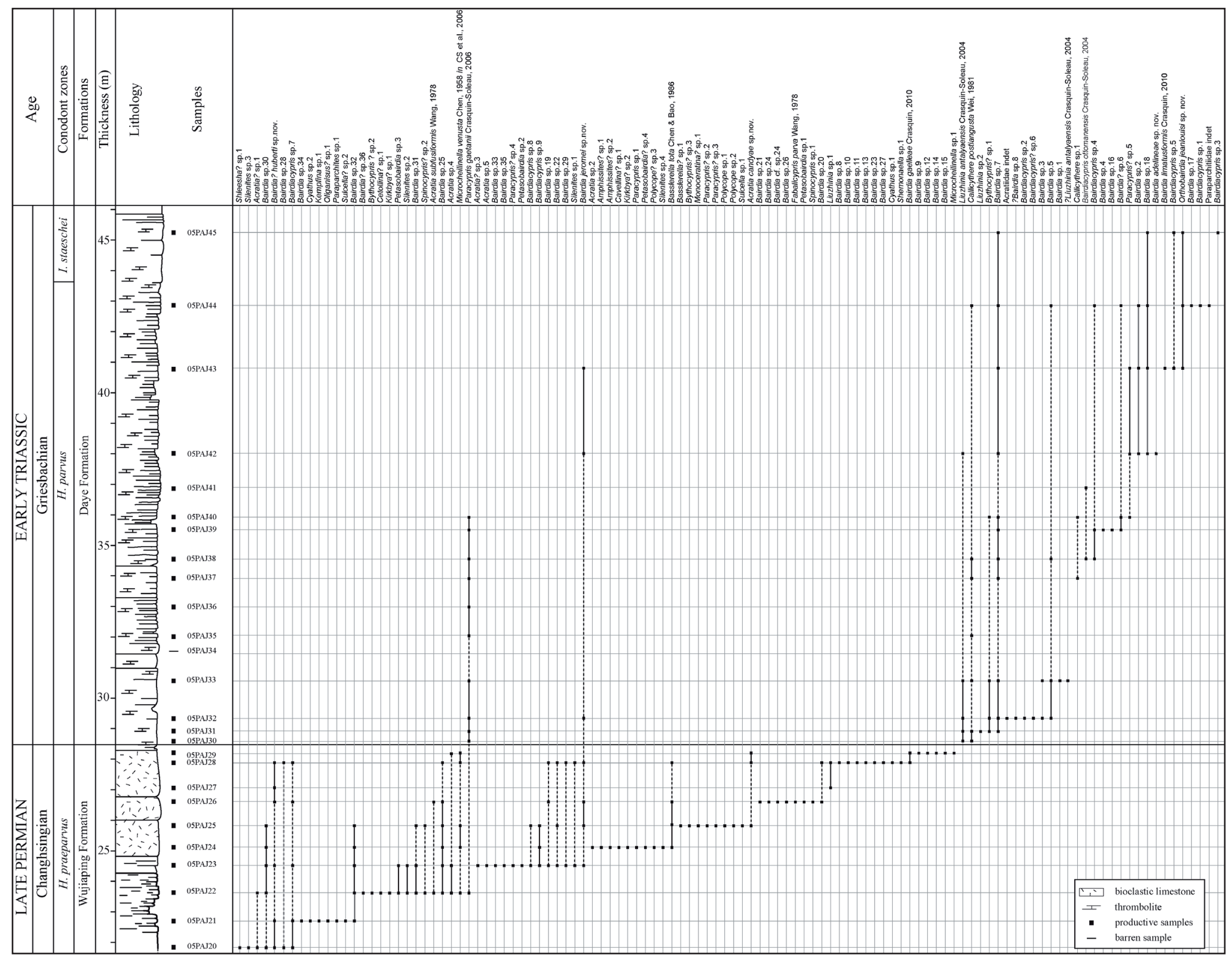

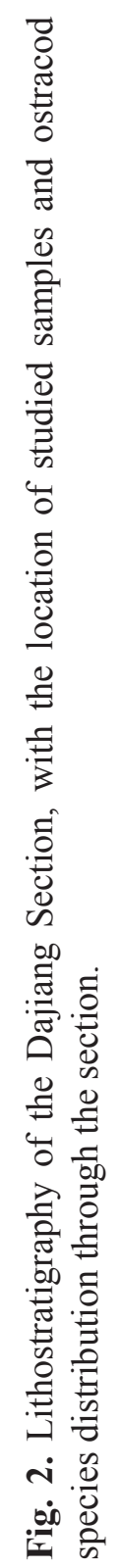




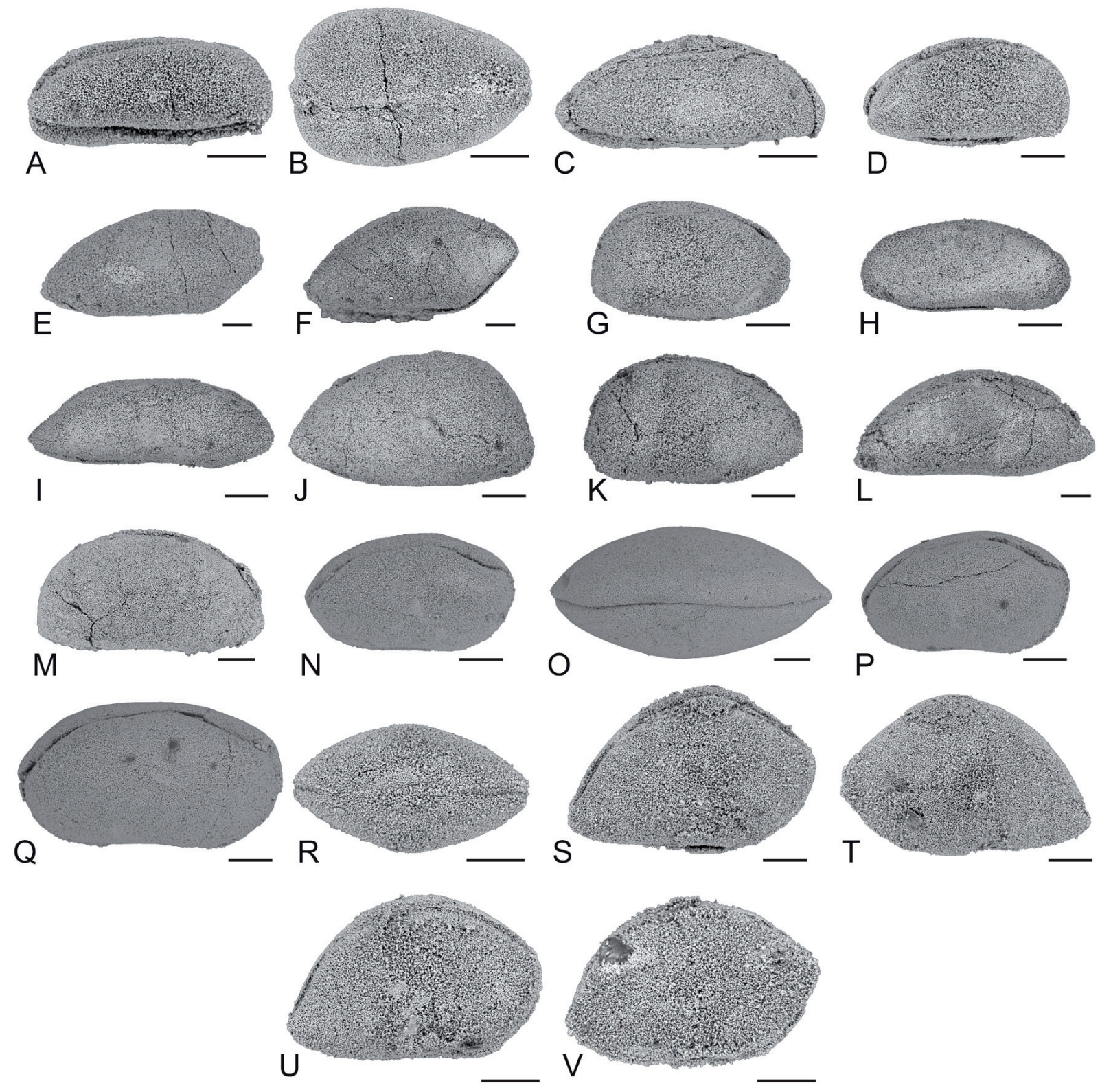

Fig. 3. Ostracods from the Dajiang section, South China. - A-D. Acratia candyae sp. nov. A. Holotype, carapace, right lateral view, P6M2917. B. Carapace, dorsal view, P6M2918. C. Paratype, carapace, right lateral view, P6M2919. D. Carapace, right lateral view, P6M2920. - E-F. Acratia subfusiformis Wang, 1978. E. Carapace, right lateral view, P6M2921. F. Carapace, right lateral view, P6M2922. G. Acratia? sp. 1, carapace, right lateral view, P6M2923. - H. Acratia? sp. 2, carapace, right lateral view, P6M2924. - I. Acratia? sp. 3, carapace, right lateral view, P6M2925. - J-K. Acratia sp. 4. J. Carapace, left lateral view, P6M2926. K. Carapace, right lateral view, P6M2927. - L. Acratia sp. 5, carapace, right lateral view, P6M2928. - M. Acratiidae indet., carapace, right lateral view, P6M2929. - N-Q. Bairdia adelineae sp. nov. N. Holotype, carapace, right lateral view, P6M2930. O. Carapace, dorsal view, P6M2931. P. paratype, carapace, right lateral view, P6M2932. Q. Carapace, right lateral view, P6M2933. - R-V. Bairdia gaelleae Crasquin, 2010. R. Carapace, dorsal view, P6M2934. S. Carapace, right lateral view, P6M2935. T. Carapace, left lateral view, P6M2936. U. Carapace, right lateral view, P6M2937. V. Carapace, right lateral view, P6M2938. - Scale $=100 \mu \mathrm{m}$. 
Roth, 1928, Basslerella Kellett, 1935 and Callicythere Wei, 1981) and 15 (genus Callicythere). Cavellinidae Egorov, 1950 (genus Sulcella Coryell \& Sample, 1932), Polycopidae Sars, 1866 (genus Polycope Sars, 1866), Aparchitidae Jones, 1901 (genus Cyathus Roth \& Skinner, 1930), Kirkbyoidea Ulrich \& Bassler, 1906 (genera Amphissites Girty, 1910, Kirkbya Jones, 1859 and Shleesha Sohn, 1961), Kloedenellidae Ulirch \& Bassler, 1908 (genus Oliganisus Geis, 1932) and Paraparchitidae Scott, 1959 (genera Paraparchites Ulrich \& Bassler, 1906 and Shemonaella Sohn, 1971) are shown in Fig. 15.

\section{Abbreviations used in the text}

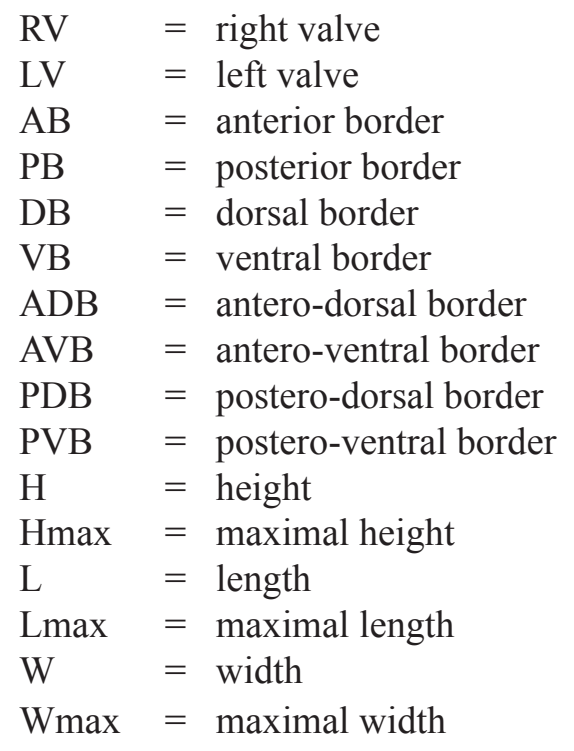

\section{Results}

\section{Taxonomic descriptions}

Class Ostracoda Latreille, 1802

Order Podocopida Müller, 1894

Sub-order Podocopina Sars, 1866

Superfamily Bairdioidea Sars, 1888

Family Acratiidae Gründel, 1962

Genus Acratia Delo, 1930

Acratia candyae sp. nov.

Fig. 3A-D

\section{Diagnosis}

Species of Acratia similar to Microcheilinella in dorsal view, with LV larger than RV and AB broadly rounded in $1 / 4$ circle.

\section{Etymology}

Personal dedication to Candy Boiteux.

\section{Material examined}

\section{Holotype}

One carapace (Fig. 3A), Dajiang section, sample 05PAJ28, collection number P6M2917. 
FOREL M.B., Ostracods from the Permian-Triassic boundary in Guizhou

Paratype

One carapace (Fig. 3C), Dajiang section, sample 05PAJ17, collection number P6M2919.

\section{Other material}

4 carapaces, several fragments. The species is known from its type locality only.

\section{Type locality}

Wujiaping Formation (Samples 05PAJ17, 25, 28, 29), Dajiang section (2533'56”N-106 39'41'”E), Guizhou Province, South China, Changhsingian, Late Permian.

\section{Measurements}

$\mathrm{L}=323-464 \mu \mathrm{m} ; \mathrm{H}=140-200 \mu \mathrm{m} ; \mathrm{H} / \mathrm{L}=0.43-0.44$.

\section{Description}

Carapace elongated and subovoid in lateral view; surface smooth; carapace slightly preplete to amplete; LV strongly overlaps RV all around the carapace; Lmax below mid-H; dorsum long and slightly rounded in both valves, angles between PDB and DB not distinct; rounded angle between DB and ADB $\left(\sim 140^{\circ}\right)$; PDB short and steep; DB long and gently bent backward; ADB short, steep and convex; ventral margin flat and straight at both valves; AVB, VB, PVB not distinct; AVB straight and slightly bent toward AB; AB broadly rounded in $1 / 4$ circle; acratian beak clear but not pronounced, located at VB; $\mathrm{PB}$ sharpen with quite large radius of curvature for the genus, maximum slightly below mid- $\mathrm{H}$; angle between AVB-AB $\left(>90^{\circ}\right)$ larger than angle between PVB-PB $\left(<90^{\circ}\right)$; dorsal view similar to the one of the genus Microcheilinella with carapace thick and Wmax located in the posterior $1 / 3$ of $\mathrm{L}$, hinge line slightly incised.

Through the ontogeny, the carapace becomes more stocky, more amplete at RV, with DB shorter and more rounded.

\section{Remarks}

The strong overlap of LV on RV all around the carapace and characteristics of dorsal view are reminiscent of the genus Microcheilinella. However the acratian beak clearly precludes Microcheilinella attribution and indicates the genus Acratia. Acratia candyae sp. nov. is very close to Acratia sp.2 sensu Crasquin, 2008 from the Late Permian of Dolomites (Crasquin et al. 2008). However, Acratia sp.2 sensu Crasquin, 2008 has longer ADB and well expressed angles between PDB, DB and ADB.

Acratia subfusiformis Wang, 1978

Figs 3E-F

Acratia subfusiformis Wang, 1978: 294-295, pl.4, figs 3-4.

Acratia subfusiformis - Shi \& Chen 1987: 49, pl.11, figs 19-22, pl. 12, figs 1-2 — Shi \& Chen 2002: 82, p1.20, figs 1-9. - Crasquin et al. 2010: 357, figs 23I-L.

? Acratia subfusiformis - Shi \& Chen 2002: 82, pl. 20, figs 12-13.

Not Acratia subfusiformis - Shi \& Chen 2002: 82, pl. 20, figs 10-11.

\section{Localities}

- Wujiaping Formation (samples 05PAJ22, 26), Dajiang section (2533'56”N-106³9’41”'E), Guizhou Province, South China, Changhsingian, Late Permian.

- Longtan Formation, Northern Guizhou and Southern Yunnan Provinces, South China, Wuchiapingian, Late Permian (Wang 1978). 
- Baoqing Member, Changxing Formation, Meishan section, Zhejiang Province, South China, Changhsingian, Late Permian (Shi \& Chen 1987).

- Matan and Pingding sections, Guangxi Province, South China Wuchiapingian and Changhsingian, Late Permian (Shi \& Chen 2002).

- Baoqing and Meishan Members, Changxing Formation, Meishan section, Zhejiang Province, South China, Changhsingian, Late Permian (Crasquin et al. 2010).

Family Bairdiidae Sars, 1887

Genus Bairdia McCoy, 1844

Bairdia adelineae sp. nov.

Fig. 3N-Q

\section{Diagnosis}

Stocky species of Bairdia with strong dorsal overlap, laterally flattened plateau-like DB, AB and PB with large radius of curvature.

\section{Etymology}

Personal dedication to Adeline Bienvenu.

\section{Material examined}

\section{Holotype}

One carapace (Fig. 3N), sample 05PAJ42, collection number P6M2930.

\section{Paratype}

One carapace (Fig. 3P), sample 05PAJ42, collection number P6M2932.

\section{Other material}

4 carapaces, several fragments. The species is known from its type locality only.

\section{Type locality}

Daye Formation (Sample 05PAJ42), Dajiang section (25³3'56”'N-106³9'41”E), Guizhou Province, South China, Griesbachian, Early Triassic.

\section{Measurements}

$\mathrm{L}=513-655 \mu \mathrm{m} ; \mathrm{H}=292-347 \mu \mathrm{m} ; \mathrm{H} / \mathrm{L}=0.53-0.58$.

\section{Description}

Carapace elongated and massive, subovoid in lateral view; surface smooth; carapace amplete to preplete; LV overlapping RV all around the carapace, dorsal overlap stronger than ventral one; dorsum subdivided into 3 distinct parts at both valves; PDB straight to slightly convex; DB straight at both valves, flattened laterally at RV; $\mathrm{ADB}$ straight to slightly concave at anterior extremity; $\mathrm{DB}$ and $\mathrm{ADB}$ longer than PDB; VB long and slightly concave in median part at RV, straight to slightly concave at LV; AVB broadly rounded and long ( $75 \%$ of Hmax); PVB straight to convex and short ( $<50 \%$ of Hmax); AB rounded with large radius of curvature, maximum in the upper $1 / 3$ of $\mathrm{H}$, bairdian beak poorly expressed but distinct; PB pointed with large radius of curvature, maximum at or slightly below mid-H; in dorsal view, carapace lenticular and thick with anterior extremity slightly tapered, Wmax located around mid-L, plateau-like laterally flattened at RV. 

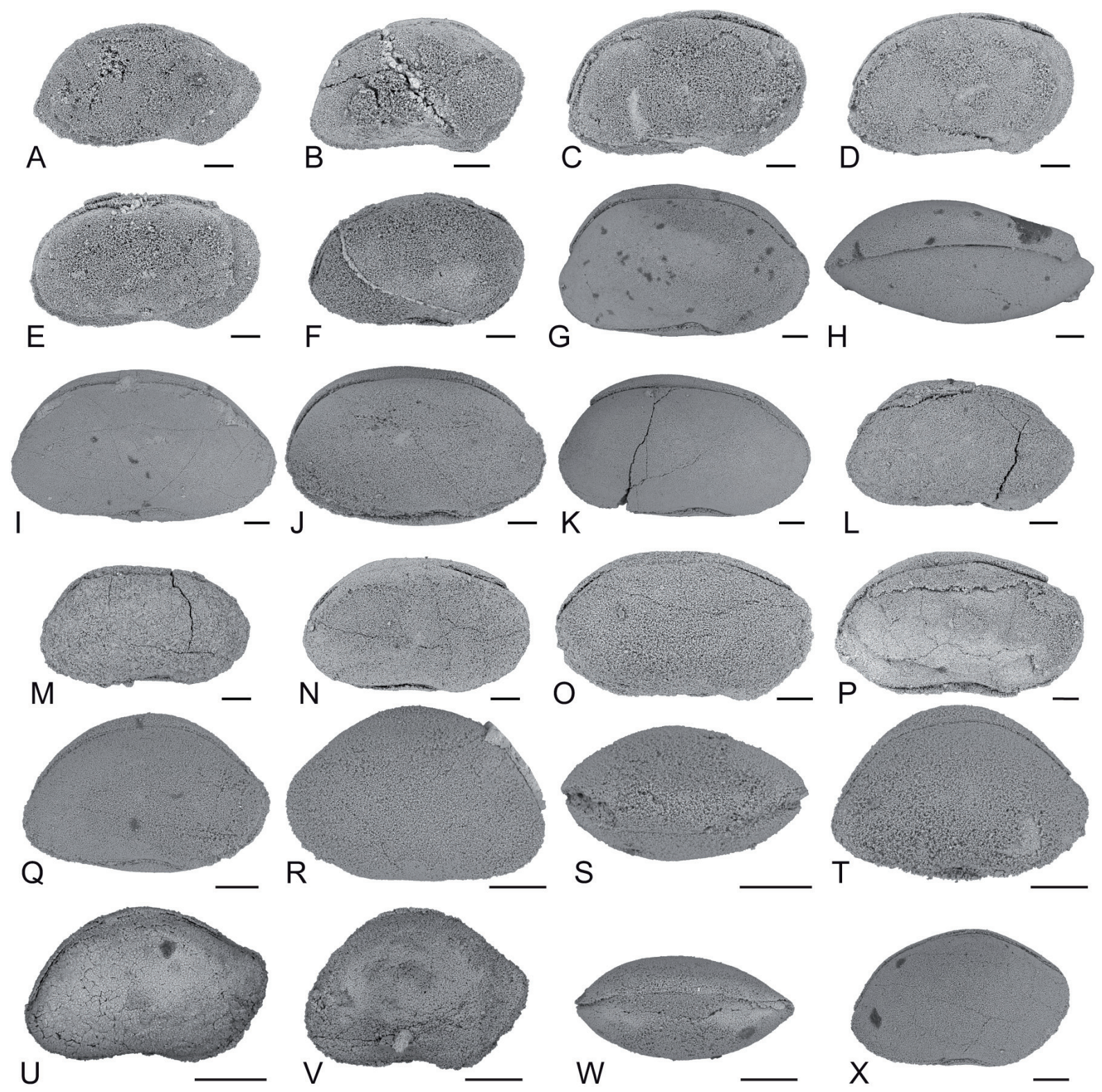

Fig. 4. Ostracods from the Dajiang section, South China. - A-F. Bairdia? huberti sp. nov. A. Carapace, right lateral view, P6M2939. B. Carapace, right lateral view, P6M2940. C. Paratype, carapace, right lateral view, P6M2941. D. Holotype, carapace, right lateral view, P6M2942. E. Carapace, right lateral view, P6M2943. F. Carapace, right lateral view, P6M2944. - G-P. Bairdia jeromei sp. nov. G. Holotype, carapace, right lateral view, P6M2945. H. Carapace, dorsal view, P6M2946. I. Carapace, right lateral view, P6M2947. J. Paratype, carapace, right lateral view, P6M2948. K. Carapace, right lateral view, P6M2949. L. Carapace, right lateral view, P6M2950. M. Carapace, right lateral view, P6M2951. N. Carapace, right lateral view, P6M2952. O. Carapace, right lateral view, P6M2953. P. Carapace, right lateral view, P6M2954. - Q-T. Bairdia limatusformis Forel, 2010. Q. Carapace, right lateral view, P6M2955. R. Carapace, left lateral view, P6M2956. S. Carapace, sub-dorsal view, P6M2957. T. Carapace, right lateral view, P6M2958. - U-V. Bairdia sp. 1. U. Carapace, right lateral view, P6M2959. V. Carapace, right lateral view, P6M2960. - W-X. Bairdia sp. 2. W. Carapace, dorsal view, P6M2961. X. Carapace, right lateral view, P6M2962. - Scale $=100 \mu \mathrm{m}$. 


\section{Remarks}

This new species can be related to Bairdia paussi Crasquin, 2010 from the Late Permian of the Meishan section, Zhejiang Province, South China (Crasquin et al. 2010). However Bairdia adelineae sp. nov. is more elongated, with stronger overlap and posterior maximum of convexity located closer to VB. It can also be compared to Orthobairdia texana (Harlton, 1927) sensu Shi \& Chen, 2002 from the Permian of Heshan and Yishan, Guangxi Province, South China (Shi \& Chen 2002). However, the bairdioid dorsal view precludes generic attribution to Orthobairdia. Bairdia adelineae sp.nov is also close to Bairdia chasae Kellett, 1934 sensu Wang, 1978 from the Late Permian of the Western Guizhou and Northeastern Yunnan Provinces, South China (Wang 1978). However the new species has a less pointed PB, a thinner ventral overlap and the dorsal view clearly shows a bairdioid shape. It is noteworthy that Bairdia chasae Kellett, 1935 sensu Wang, 1978 seems wrongly attributed to the genus Bairdia: the dorsal view shows a typical orthobairdian shape.

Bairdia gaelleae Crasquin, 2010

Fig. 3R-V

Bairdia gaelleae Crasquin, 2010: 344-346, figs 13W-Z.

? Bairdia hassi - Shi \& Chen 2002: 66, pl. 4, figs 11-15.

\section{Localities}

- Wujiaping Formation (samples 05PAJ, 28, 29), Dajiang section (N25³3'56”-E106³9’41”), Guizhou Province, South China, Changhsingian, Late Permian.

- Matan and Pingding sections, Guangxi Province, South China, Wuchiapingian, Late Permian (Shi \& Chen 2002).

- Baoqing and Meishan members, Changxing Formation, Meishan section, Zhejiang Province, South China, Changhsingian, Late Permian (Crasquin et al. 2010).

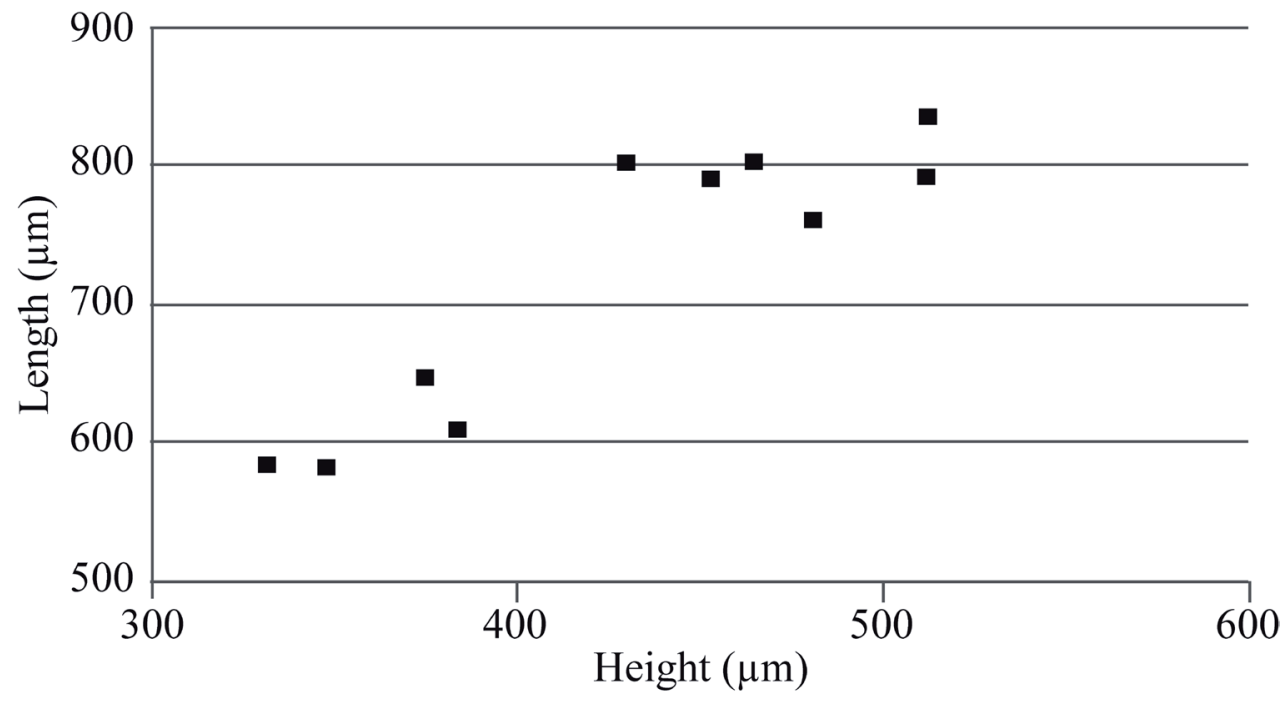

Fig. 5. Height/length diagram of Bairdia ?huberti sp. nov. 
Bairdia? huberti sp. nov.

Fig. 4A-F

\section{Diagnosis}

Species attributed with doubt to the genus Bairdia, with relatively elongated carapace, ADB and DB not distinct, very large AB.

\section{Etymology}

Personal dedication to Hubert Colas.

\section{Material examined}

\section{Holotype}

One carapace (Fig. 4D), sample 05PAJ28, collection number P6M2942.

\section{Paratype}

One carapace (Fig. 4C), sample 05PAJ28, collection number P6M2941.

\section{Other material}

10 carapaces, several fragments. The species is known from its type locality only.

\section{Type locality}

Wujiaping Formation (samples 05PAJ20, 21, 23, 26-28), Dajiang section (2533'56”N-106 39'41'”E), Guizhou Province, South China, Changhsingian, Late Permian.

\section{Measurements (Fig. 5)}

$\mathrm{L}=581-837 \mu \mathrm{m} ; \mathrm{H}=332-512 \mu \mathrm{m} ; \mathrm{H} / \mathrm{L}=0.53-0.64$.

\section{Description}

Carapace relatively elongated, ovoid in lateral view; PDB long, straight to slightly convex; PDB-DB angle around $110^{\circ}$; $\mathrm{DB}$ and $\mathrm{ADB}$ not clearly distinct and along relative constant $\mathrm{H}$, close to Hmax; VB concave; AVB rounded and long ( $\sim 75 \%$ of Hmax); PVB very short and rounded; AB with large radius of curvature, maximum located on the upper $1 / 4$ of $\mathrm{H}$; $\mathrm{PB}$ with narrow radius of curvature, maximum located in the lower $1 / 4$ of $\mathrm{H}$; Lmax located around mid-H.

\section{Remarks}

High intraspecific variations that mainly affect the $\mathrm{ADB}$ and $\mathrm{AB}$ are observed in Bairdia? huberti sp. nov. They create continuum between Cryptobairdia-like (ABD not distinct: Fig. 4C-E) and Bairdialike specimens (ABD differentiated: Fig. 4A, B, F). The new species is therefore attributed with doubt to the genus Bairdia. Bairdia? huberti sp. nov. is similar to Bairdia broutini Crasquin, 2010 from the Late Permian of the Meishan section, Zhejiang Province, South China (Crasquin et al. 2010) because of the relative constant and high $\mathrm{H}$. However, the new species is more elongated, with longer DB.

\section{Bairdia jeromei sp. nov.}

Fig. 4G-P

\section{Diagnosis}

Massive and subrectangular species of Bairdia, with DB and VB subparallel, long DB ( $\sim 60 \%$ of Lmax), rhombic dorsal view. 


\section{Etymology}

Personal dedication to Jérôme Cougoul.

\section{Material examined}

\section{Holotype}

One carapace (Fig. 4G), sample 05PAJ43, collection number P6M2945.

\section{Paratype}

One carapace (Fig. 4J), sample 05PAJ43, collection number P6M2948.

\section{Other material}

10 carapaces, several fragments. The species is known from its type locality only.

\section{Type locality}

Wujiaping and Daye Formations (samples 05PAJ23, 25, 26, 28, 32, 42, 43), Dajiang section (253'56'N-106 $39^{\circ} 41^{\prime \prime}$ E), Guizhou Province, South China, Changhsingian and Griesbachian, Late Permian and Early Triassic.

\section{Measurements (Fig. 6)}

$\mathrm{L}=722-1024 \mu \mathrm{m} ; \mathrm{H}=410-597 \mu \mathrm{m} ; \mathrm{H} / \mathrm{L}=0.56-0.64$.

\section{Description}

Carapace massive, elongated and subrectangular in lateral view; surface smooth; LV larger than RV, overlapping at dorsum and VB; bairdian beaks at $\mathrm{AB}$ and $\mathrm{PB}$ poorly expressed; $\mathrm{PDB}, \mathrm{DB}$ and $\mathrm{ADB}$ distinct at both valves; PDB steeply bent backward, long ( $65 \%$ of Hmax) and slightly convex; DB long ( $\sim 60 \%$ of Lmax) and gently convex; ADB slightly concave at RV, shorter than PDB; ventral

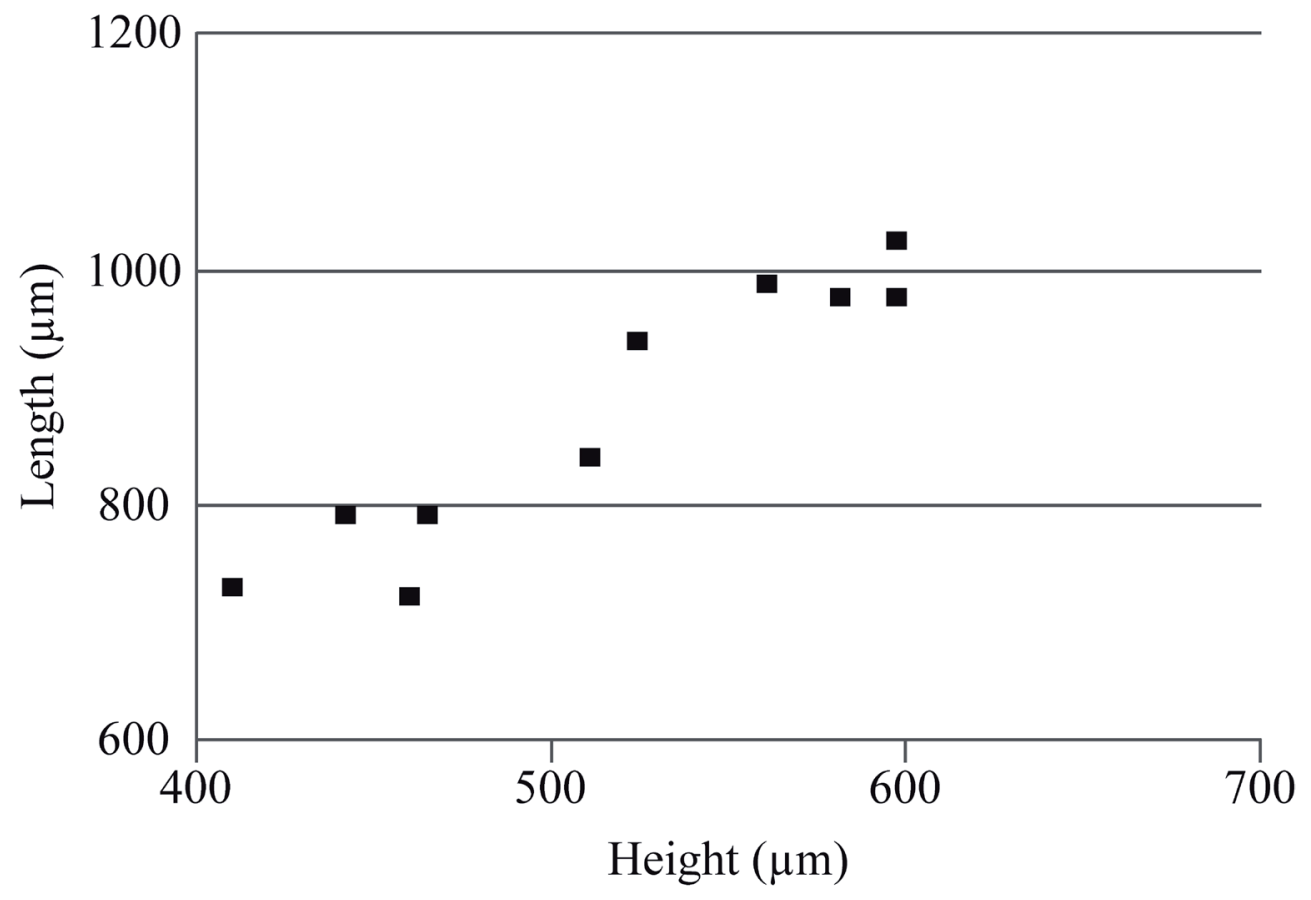

Fig. 6. Height/length diagram of Bairdia jeromei sp. nov. 
part rounded; VB long, convex at LV, concave at RV; AVB and PVB rounded, AVB longer than PVB; carapace slightly postplete to amplete; Lmax below mid-H; dorsal view rhombic with LV's Wmax in posterior $1 / 3$ of Lmax, in anterior $1 / 3$ at RV.

\section{Remarks}

In lateral view, Bairdia jeromei sp. nov. is similar to Orthobairdia sp.1 sensu Crasquin et al., 2010 from the Permian of the Meishan section, Zhejiang Province, South China (Crasquin et al. 2010) but here the dorsal view clearly precludes the generic attribution to Orthobairdia.

Bairdia limatusformis Forel, 2010

Fig. 4Q-T

Silenites limatus - Shi \& Chen 1987: 62, pl.15, figs 15-19.

Bairdia limatusformis - Crasquin et al. 2010: 346-347, figs 9E-G.

Not Silenites limatus - Shi \& Chen 2002: 95, pl.27, figs 2-17.

\section{Localities}

- Daye Formation (sample 05PAJ43), Dajiang section (2533'56”N-106³9'41'E), Guizhou Province, South China, Griesbachian, Early Triassic.

- Baoqing and Meishan members, Changxing formation, Meishan section, Zhejiang Province, South China, Changhsingian, Late Permian (Shi \& Chen 1987; Crasquin et al. 2010).

- Matan and Pingding sections, Guangxi Province, South China, Wuchiapingian, Late Permian (Shi \& Chen 2002).

Genus Bairdiacypris Bradfield, 1935

Bairdiacypris ottomanensis Crasquin-Soleau, 2004

Fig. 10E-H

Bairdiacypris ottomanensis Crasquin-Soleau, 2004: 285-286, pl. 2, figs 13-24.

Bairdiacypris ottomanensis - Crasquin-Soleau \& Kershaw 2005: pl. I, figs 10-12. - Forel et al. 2009: 819, fig. 4 (1).

\section{Localities}

- Daye Formation (samples 05PAJ38, 41), Dajiang section $\left(25^{\circ} 33^{\prime} 56^{\prime \prime} \mathrm{N}-106^{\circ} 39^{\prime} 41^{\prime \prime} \mathrm{E}\right)$, Guizhou Province, South China, Griesbachian, Early Triassic (Forel et al. 2009; this study).

- Kokarkuyu Formation, Çürük dağ section, Western Taurus, Antalya Nappes, Turkey, Early Triassic (Crasquin-Soleau et al. 2004).

- Feixianguan Formation, Laolongdong section, Sichuan Province, South China, Induan, Early Triassic (Crasquin \& Kershaw 2005).

Genus Fabalicypris Brady, 1880

Fabalicypris parva Wang, 1978

Fig. 11I

Fabalicypris parva Wang, 1978: 293, pl. 2, figs 12a-b, 13a-b.

Fabalicypris hungarica Kozur, 1985: 82, pl. 2, figs 2, 9, 10.

Bairdiacypris opulenta - Shi \& Chen 1987: 51, pl. 13, fig. 10. 

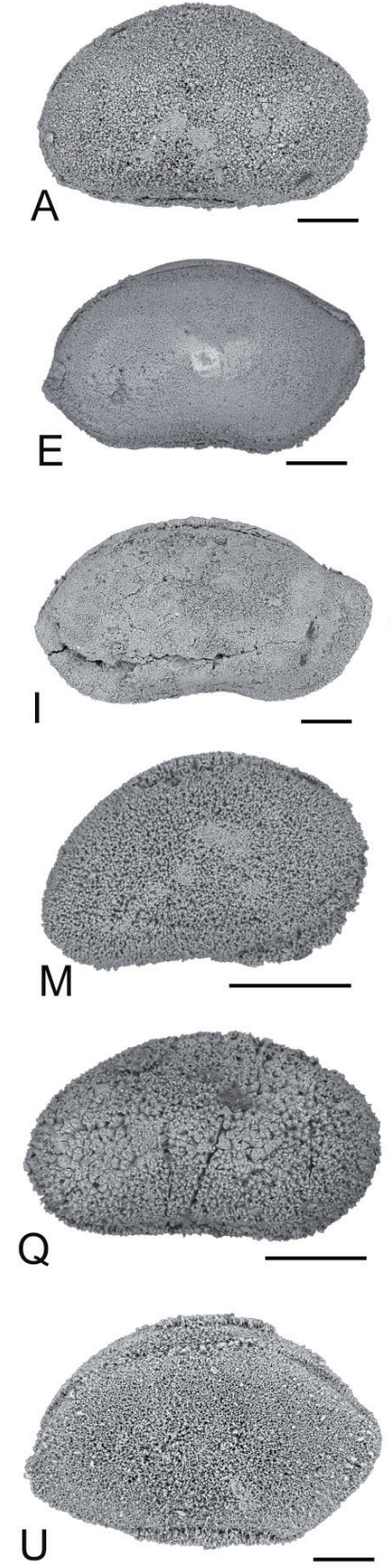
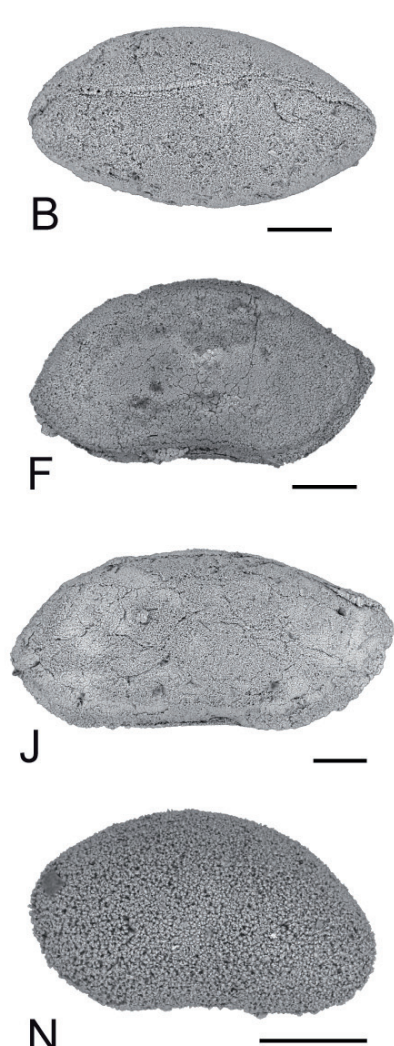

N
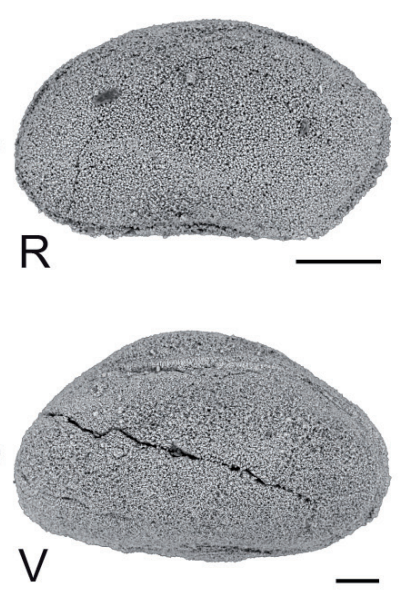

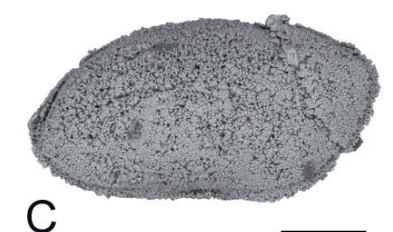

C
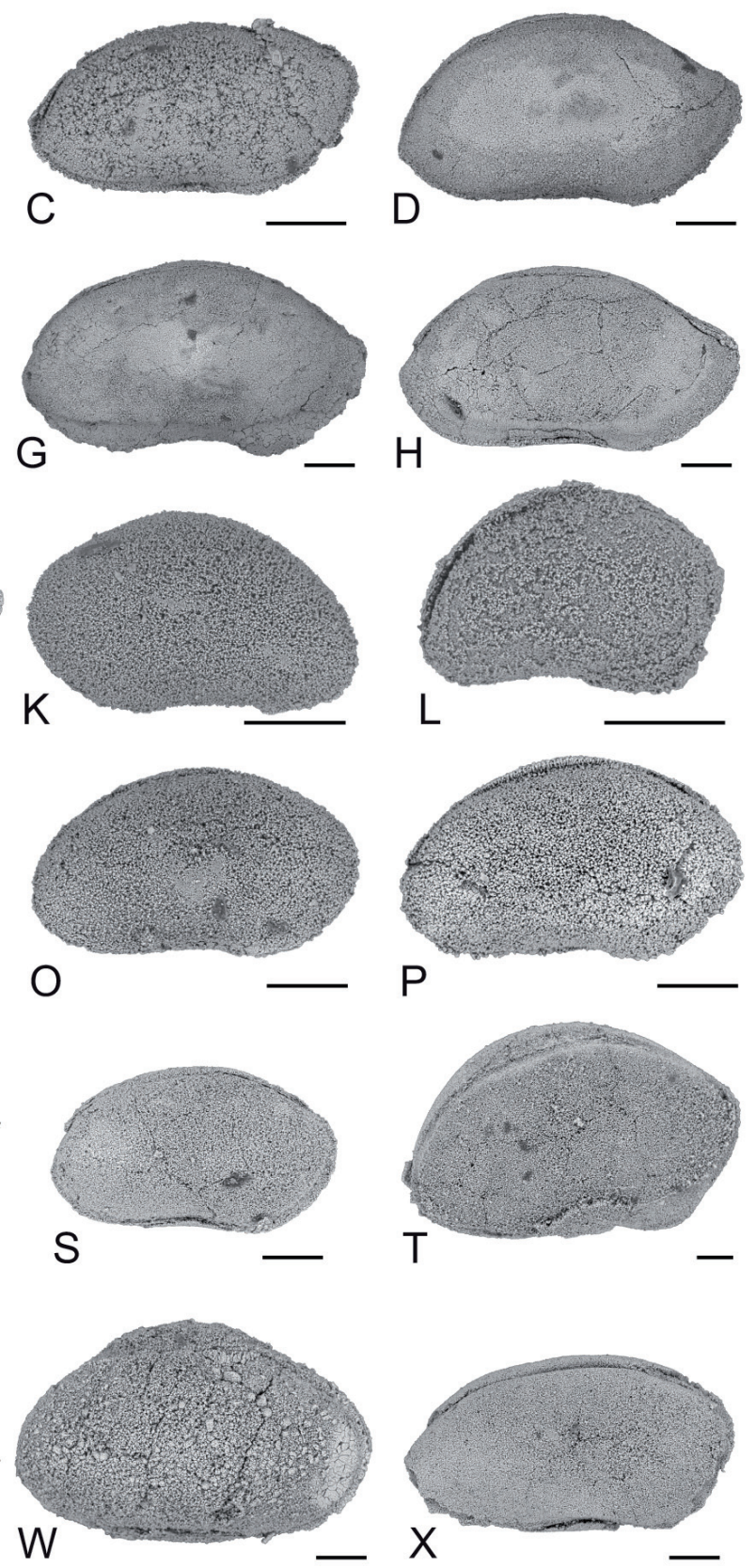

Fig. 7. Ostracods from the Dajiang section, South China. - A-B. Bairdia sp. 3. A. Carapace, right lateral view, P6M2963. B. Carapace, dorsal view, P6M2964. - C. Bairdia sp. 4, Carapace, right lateral view, P6M2965. - D-J. Bairdia sp. 5. D. Carapace, right lateral view, P6M2966. E. Carapace, right lateral view, P6M2967. F. Carapace, right lateral view, P6M2968. G. Carapace, right lateral view, P6M2969. H. Carapace, right lateral view, P6M2970. I. Carapace, right lateral view, P6M2971. J. Carapace, right lateral view, P6M2972. - K-M. Bairdia? sp. 6. K. Carapace, left lateral view, P6M2973. L. Carapace, right lateral view, P6M2974. L. Carapace, right lateral view, P6M2975. - N-S. Bairdia sp. 7. N. Carapace, left lateral view, P6M2976. O. Carapace, right lateral view, P6M2977. P. Carapace, right lateral view, P6M2978. Q. Carapace, right lateral view, P6M2979. R. Carapace, right lateral view, P6M2980. S. Carapace, right lateral view, P6M2981. - T-U. Bairdia sp. 8. T. Carapace, right lateral view, P6M2982. U. Carapace, right lateral view, P6M2983. - V-W. ?Bairdia sp. 8. V. Carapace, right lateral view, P6M2984. W. Carapace, right lateral view, P6M2985. - X. Bairdia sp. 9, carapace, right lateral view, P6M2986. - Scale $=100 \mu \mathrm{m}$. 

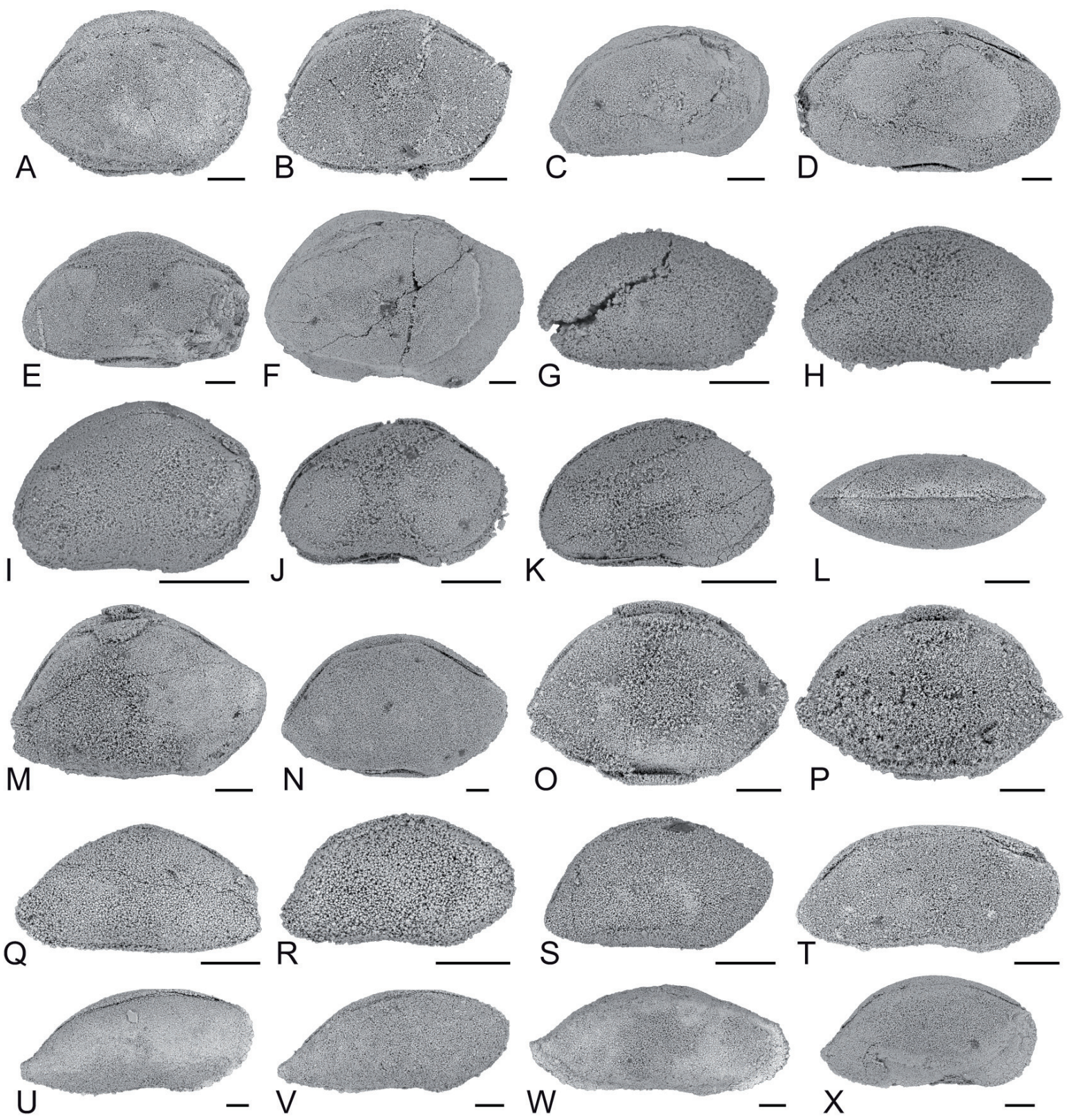

Fig. 8. Ostracods from the Dajiang section, South China. - A. Bairdia sp. 10, carapace, right lateral view, P6M2987. - B. Bairdia sp. 11, carapace, right lateral view, P6M2988. - C. Bairdia sp. 12, carapace, right lateral view, P6M2989. - D. Bairdia sp. 13, carapace, right lateral view, P6M2990. E. Bairdia sp. 14, carapace, right lateral view, P6M2991. - F. Bairdia sp. 15, carapace, right lateral view, P6M2992. - G. Bairdia sp. 16, carapace, right lateral view, P6M2993. - H. Bairdia sp. 17, carapace, right lateral view, P6M2994. - I-L. Bairdia sp. 18. I. Carapace, right lateral view, P6M2995. J. Carapace, right lateral view, P6M2996. K. Carapace, right lateral view, P6M2997. L. Carapace, dorsal view, P6M2998. - M-N. Bairdia sp. 19. M. Carapace, right lateral view, P6M2999. N. Carapace, right lateral view, P6M3000. - O-P. Bairdia sp. 20. O. Carapace, right lateral view, P6M3001. P. Carapace, right lateral view, P6M3002. - Q. Bairdia sp. 21, carapace, right lateral view, P6M3003. R-S. Bairdia sp. 22. R. Carapace, right lateral view, P6M3004. S. Carapace, right lateral view, P6M3005. - T. Bairdia sp. 23, carapace, right lateral view, P6M3006. - U-W. Bairdia sp. 24. U. Carapace, right lateral view, P6M3007. V. Carapace, right lateral view, P6M3008. W. Carapace, right lateral view, P6M3009. - X. Bairdia cf. sp. 24, carapace, right lateral view, P6M3010. - Scale $=100 \mu \mathrm{m}$. 

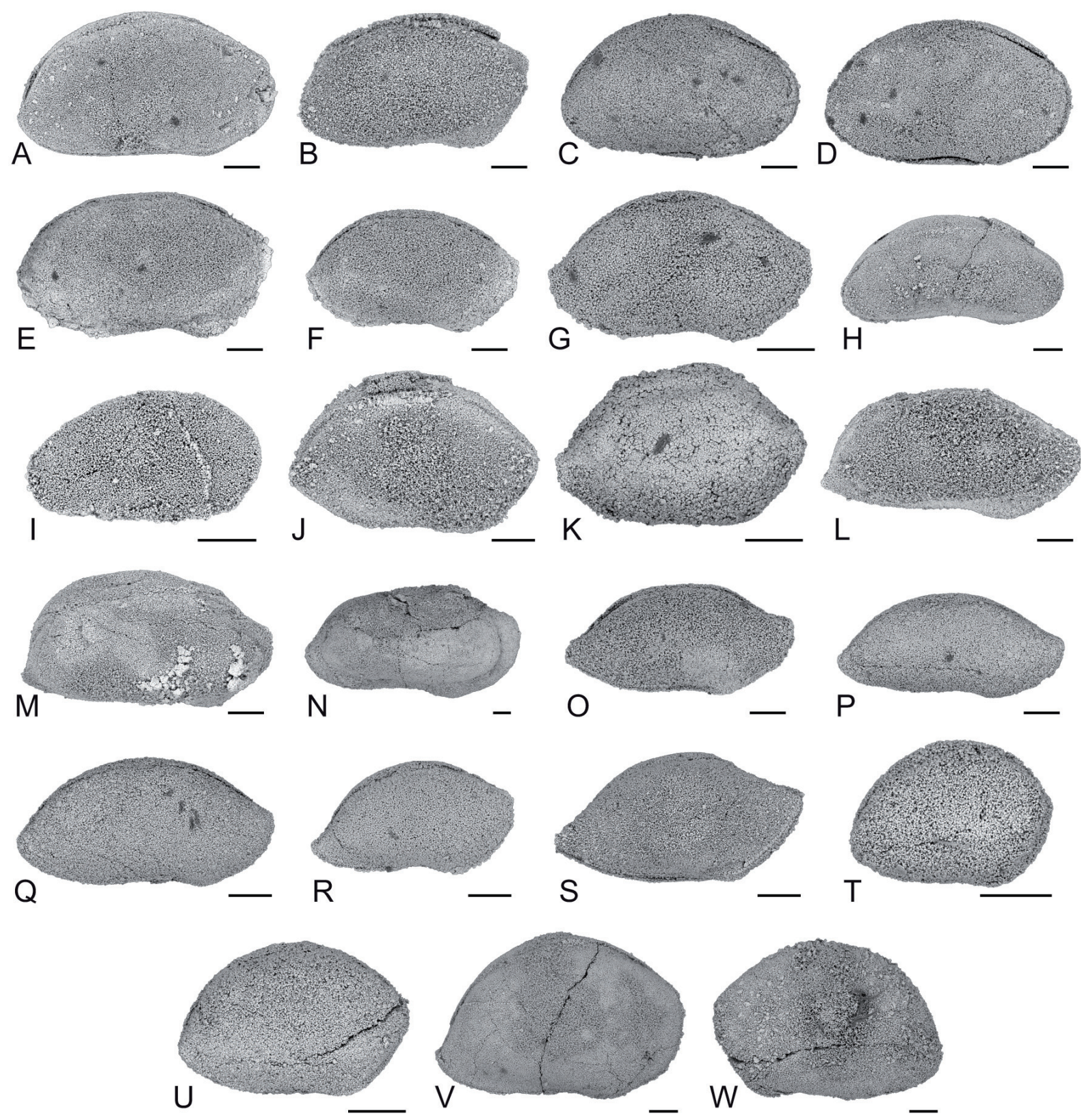

Fig. 9. Ostracods from the Dajiang section, South China. - A-D. Bairdia sp. 25. A. Carapace, right lateral view, P6M3011. B. Carapace, right lateral view, P6M3012. C. Carapace, right lateral view, P6M3013. D. Carapace, right lateral view, P6M3014. - E-G. Bairdia sp. 26. E. Carapace, right lateral view, P6M3015. F. Carapace, right lateral view, P6M3016. G. Carapace, right lateral view, P6M3017. - H-I. Bairdia sp. 27. H. Carapace, right lateral view, P6M3018. I. Carapace, right lateral view, P6M3019. - J-K. Bairdia sp. 28. J. Carapace, right lateral view, P6M3020. K. Carapace, right lateral view, P6M3021. - L-N. Bairdia sp. 29. L. Carapace, right lateral view, P6M3022. M. Carapace, right lateral view, P6M3023. N. Carapace, right lateral view, P6M3024. - O-Q. Bairdia sp. 30. O. Carapace, right lateral view, P6M3025. P. Carapace, right lateral view, P6M3026. Q. Carapace, right lateral view, P6M3027. - R-S. Bairdia sp. 31. R. Carapace, right lateral view, P6M3028. S. Carapace, right lateral view, P6M3029. - T-W. Bairdia sp. 32. T. Carapace, right lateral view, P6M3030. U. Carapace, right lateral view, P6M3031. V. Carapace, right lateral view, P6M3032. W. Carapace, left lateral view, P6M3033. - Scale $=100 \mu \mathrm{m}$. 

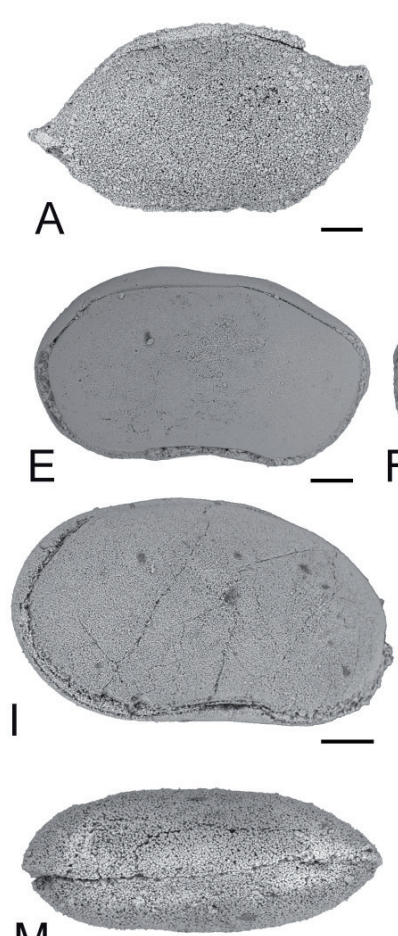

M
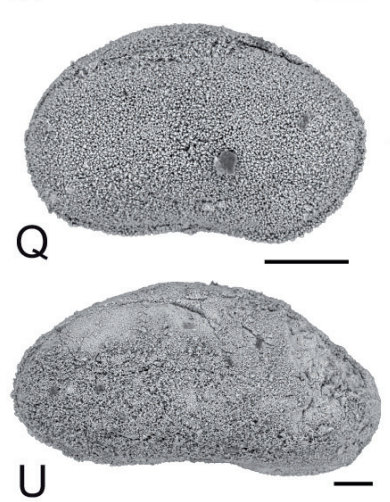
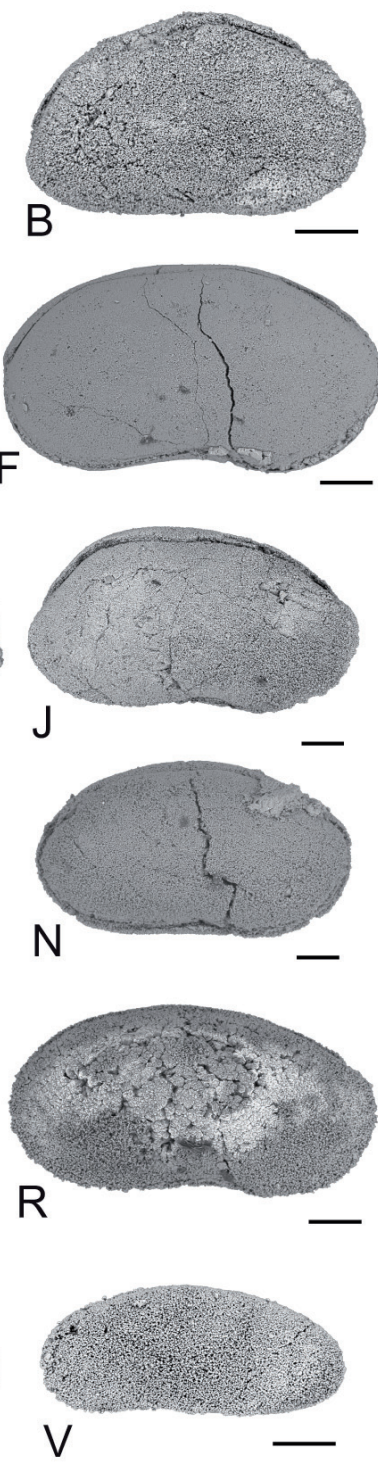
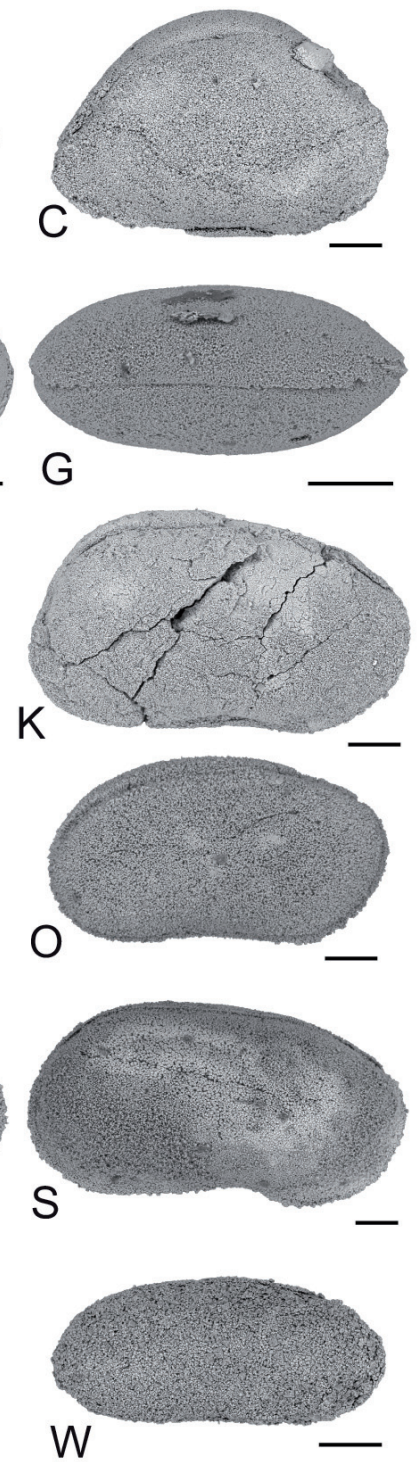

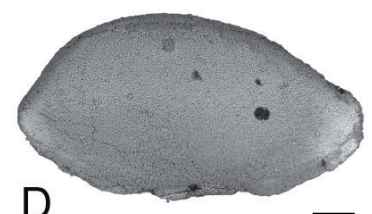

$\mathrm{D}$

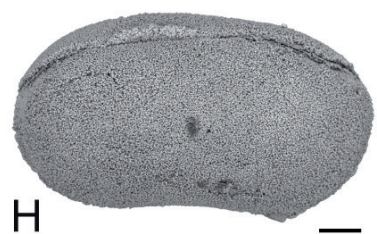

$\mathrm{H}$
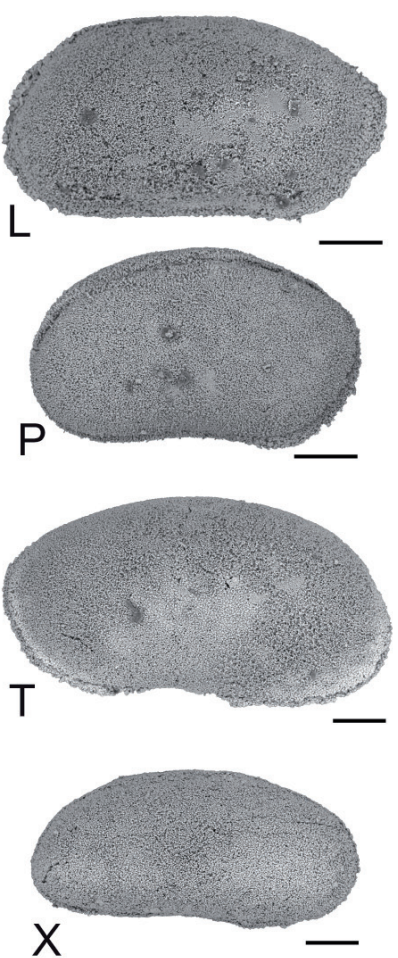

Fig. 10. Ostracods from the Dajiang section, South China. - A. Bairdia sp. 33, carapace, right lateral view, P6M3034. - B. Bairdia sp. 34, carapace, right lateral view, P6M3035. - C. Bairdia sp. 35, carapace, right lateral view, P6M3036. - D. Bairdia? sp. 36, carapace, right lateral view, P6M3037. E-H. Bairdiacypris ottomanensis Crasquin-Soleau, 2004. E. Carapace, right lateral view, P6M3038. F. Carapace, right lateral view, P6M3039. G. Carapace, dorsal view, P6M3040. H. Carapace, right lateral view, P6M3041. - I. Bairdiacypris sp. 1, carapace, right lateral view, P6M3042. - J-K. Bairdiacypris sp. 2. J. Carapace, right lateral view, P6M3043. K. Carapace, right lateral view, P6M3044. - L-M. Bairdiacypris sp. 3. L. Carapace, right lateral view, P6M3045. M. Carapace, dorsal view, P6M3046. N-Q. Bairdiacypris sp. 4. N. Carapace, right lateral view, P6M3047. O. Carapace, right lateral view, P6M3048. P. Carapace, right lateral view, P6M3049. Q. Carapace, right lateral view, P6M3050. R-T. Bairdiacypris sp. 5. R. Carapace, right lateral view, P6M3051. S. Carapace, right lateral view, P6M3052. T. Carapace, left lateral view, P6M3053. - U. Bairdiacypris? sp. 6, carapace, right lateral view, P6M3054. - V-X. Bairdiacypris sp. 7. V. Carapace, right lateral view, P6M3055. W. Carapace, right lateral view, P6M3056. X. Carapace, right lateral view, P6M3057. - Scale $=100 \mu \mathrm{m}$. 

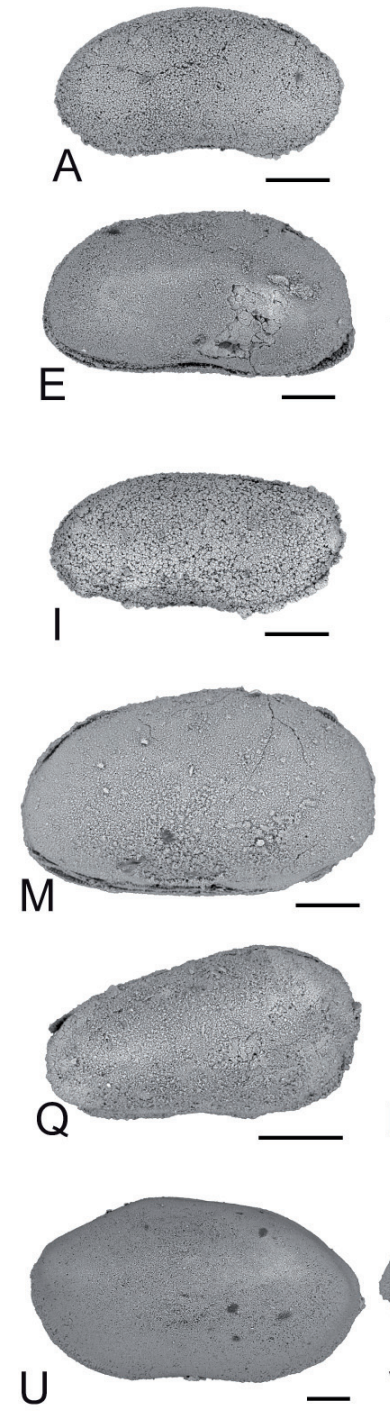
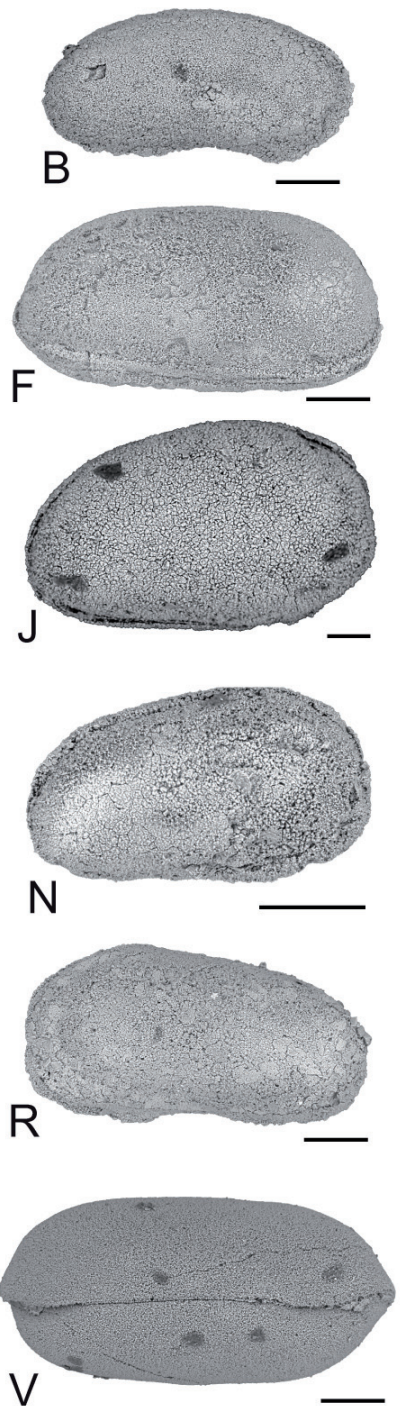
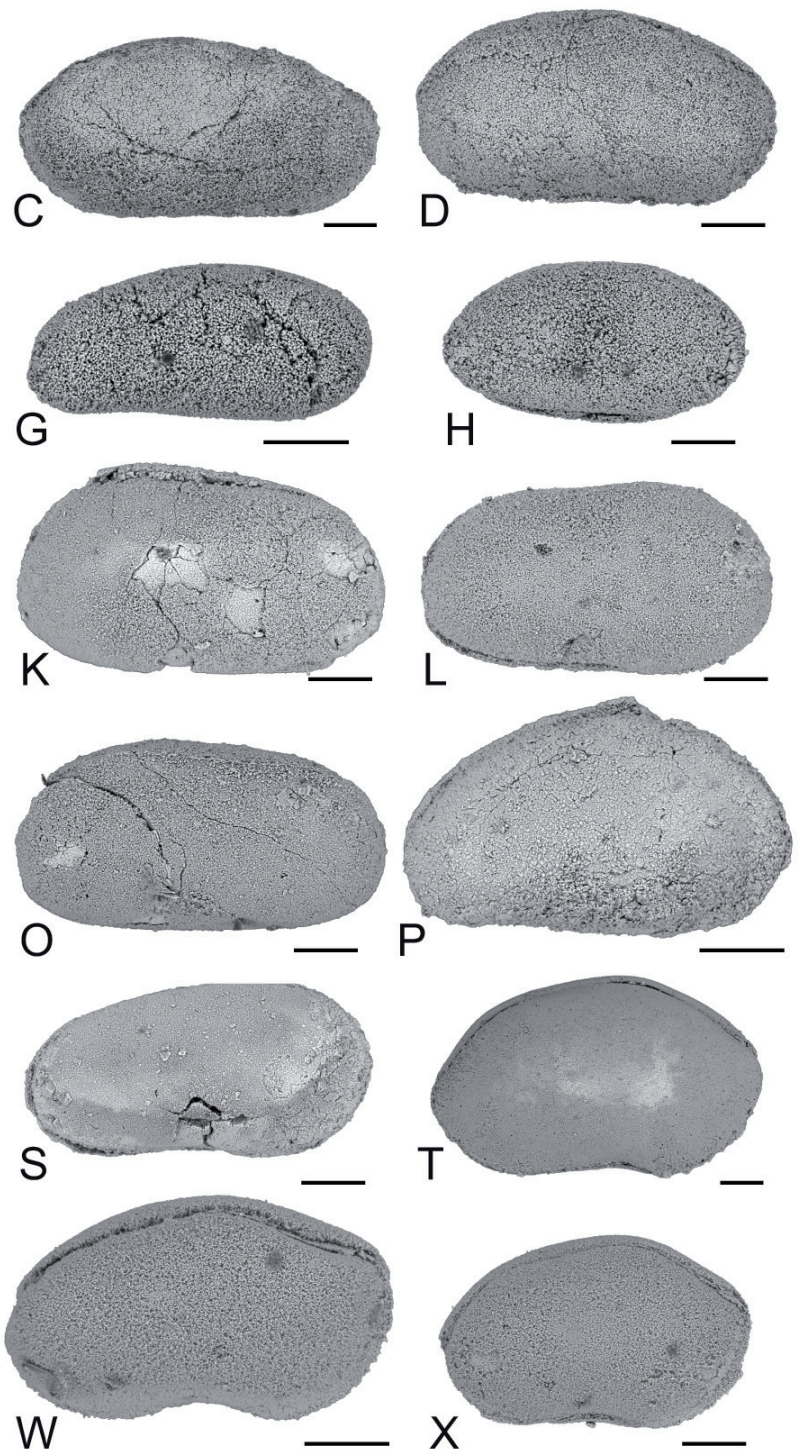

Fig. 11. Ostracods from the Dajiang section, South China. - A-B. Bairdiacypris sp. 8. A. Carapace, right lateral view, P6M3058. B. Carapace, right lateral view, P6M3059. - C-D. Bairdiacypris sp. 9. C. Carapace, right lateral view, P6M3060. D. Carapace, right lateral view, P6M3061 - - E-F. Bythocypris? sp. 1. E. Carapace, right lateral view, P6M3062. F. Carapace, right lateral view, P6M3063. - G. Bythocypris sp. 2, carapace, right lateral view, P6M3064. - H. Bythocypris? sp. 3, carapace, right lateral view, P6M3065. - I. Fabalicypris parva Wang, 1978, carapace, right lateral view, P6M3066. - J-N. Liuzhinia antalyaensis Crasquin-Soleau, 2004. J. Carapace, right lateral view, P6M3067. K. Carapace, left lateral view, P6M3068. L. Carapace, left lateral view, P6M3069. M. Carapace, right lateral view, P6M3070. N. Carapace, right lateral view, P6M3071. - O. ?Liuzhinia antalyaensis CrasquinSoleau, 2004, carapace, left lateral view, P6M3072. - P. Liuzhinia sp., carapace, right lateral view, P6M3073. - Q-S. Liuzhinia sp. 2. Q. Carapace, right lateral view, P6M3074. R. Carapace, left lateral view, P6M3075. S. Carapace, right lateral view, P6M3076. - T-X. Orthobairdia jeanlouisi sp. nov. T. Holotype, carapace, right lateral view, P6M3077. U. Carapace, left lateral view, P6M3078. V. Paratype, carapace, dorsal view, P6M3079. W. Carapace, right lateral view, P6M3080. X. Carapace, right lateral view, P6M3081. - Scale $=100 \mu \mathrm{m}$. 
Fabalicypris parva - Crasquin-Soleau et al. 2004: 286, pl. 3, figs 4-5. - Mette 2008: pl. 2, fig. 8 Crasquin et al. 2010: 353, fig. 9A'-B'.

\section{Localities}

- Wujiaping Formation (sample 05PAJ26), Dajiang section (25³3'56”N-106³9’41”E), Guizhou Province, South China, Changhsingian, Late Permian.

- Longtan and Changxing Formations, Guizhou and Yunnan Provinces, South China, Wuchiapingian and Changhsingian, Late Permian (Wang 1978).

- Bükk Mountains, Hungary, Late Moscovian, Carboniferous, Late Permian (Kozur 1985).

- Changxing formation, Meishan section, Zhejiang Province, South China, Changhsingian, Late Permian (Shi \& Chen 1987; Crasquin et al. 2010).

- Çürük dağ section, Western Taurus, Turkey, Wuchiapingian and Changhsingian, Late Permian (Crasquin-Soleau et al. 2004).

- Zal section, Iran, Changhsingian, Late Permian (Mette 2008).

\section{Remarks}

According to previously published records of Fabalicypris parva Wang, 1978, this species is known from the Late Moscovian (Carboniferous; Kozur 1985) to the Changhsingian (Late Permian; Wang 1978; Shi \& Chen 1987; Crasquin-Soleau et al. 2004; Mette 2008; Crasquin et al. 2010). This long stratigraphic range is suspicious: most of the occurrences being late Permian in age, the Carboniferous one seems questionable. When defining $F$. hungarica Kozur, 1985 as a synonym of $F$. parva Wang, 1978, Crasquin et al. (2010) stated that $F$. hungarica had a more rounded PB and a maximum of convexity located higher than F. parva. However, because all intermediate shapes between the 2 species were available, they considered all the specimens as belonging to the same species. This observation together with the long stratigraphic repartition of the species seems to indicate an evolutionary trend within one single species. More material is necessary to test this hypothesis.

Genus Liuzhinia Zheng, 1976

Liuzhinia antalyaensis Crasquin-Soleau, 2004

Fig. 11J-N

Liuzhinia antalyaensis Crasquin-Soleau, 2004: 286, pl. 3, figs 6-13.

Liuzhinia antalyaensis - Crasquin-Soleau et al. 2006: 62, pl. 3, figs 12-13. - Crasquin et al. 2008: 249, pl. 4, figs 9, 12 .

\section{Localities}

- Samples 05PAJ30-33, 42, Daye Formation, Dajiang section (25³3'56”N-106³9'41'"E), Guizhou Province, South China, Griesbachian, Early Triassic.

- Kokarkuyu Formation, Çürük dağ section, Western Taurus, Turkey, Early Triassic (Crasquin-Soleau et al. 2004).

- Jinya / Waili section, Fengshan area, Guangxi Province, South China, Griesbachian, Early Triassic (Crasquin-Soleau et al. 2006).

- Bulla section, Dolomites, Southern Alps, Northern Italy, Early Triassic (Crasquin et al. 2008). 
Genus Orthobairdia, Sohn, 1960

Orthobairdia jeanlouisi sp. nov.

Fig. 11T-X

\section{Diagnosis}

Species of Orthobairdia with preplete carapace, sharp angles between PDB, DB and ADB $\left(\sim 150^{\circ}\right)$ and large radius of curvature at $\mathrm{AB}$ and $\mathrm{PB}$.

\section{Etymology}

Personal dedication to Jean-Louis Forel.

\section{Material examined}

\section{Holotype}

One carapace (Fig. 11T), sample 05PAJ43, collection number P6M3077.

\section{Paratype}

One carapace (Fig. 11V), sample 05PAJ43, collection number P6M3079.

\section{Other material}

13 carapaces, several fragments. The species is known from its type locality only.

\section{Type locality}

Daye Formation (samples 05PAJ43-45), Dajiang section (253'5'”N-106³9'41'”E), Guizhou Province, South China, Griesbachian, Early Triassic.

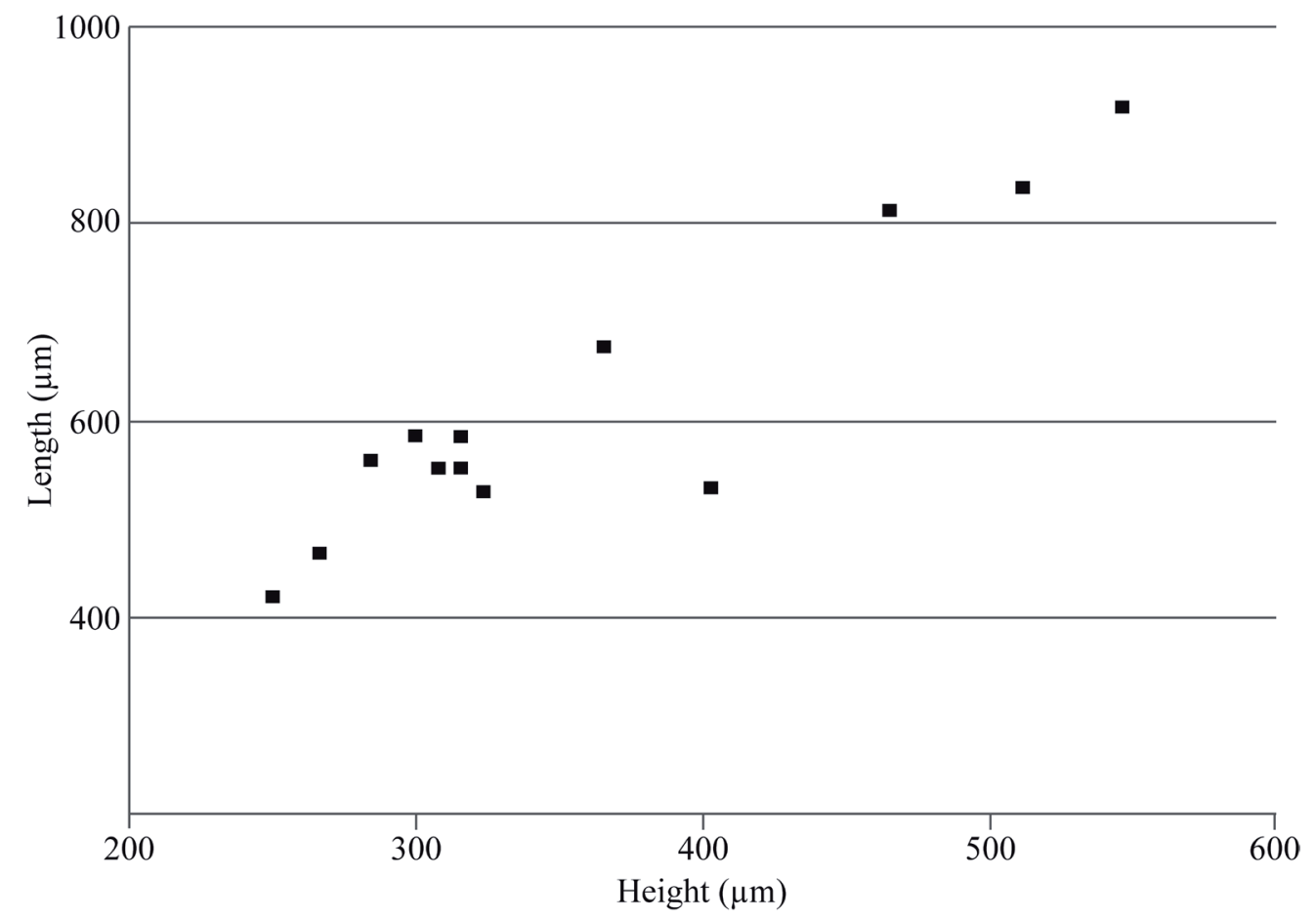

Fig. 12. Height/length diagram of Orthobairdia jeanlouisi sp. nov. 


\section{Measurements (Fig. 12)}

$\mathrm{L}=422-918 \mu \mathrm{m} ; \mathrm{H}=250-546 \mu \mathrm{m} ; \mathrm{H} / \mathrm{L}=0.50-0.75$.

\section{Description}

Carapace subtriangular in lateral view; surface smooth; LV overlapping RV all around the carapace, dorsal overlap thicker; angles between $\mathrm{PDB}, \mathrm{DB}$ and $\mathrm{ADB} \sim 150^{\circ}$ at both valves; PDB straight with steep backward slope; DB and ADB long, together $~ 60 \%$ of Lmax; DB straight at both valves, bent backward; ADB straight at LV, slightly convex at RV, less steep than PDB; VB concave; AVB long and gently rounded; PVB shorter, gently rounded; AB subtriangular, with large radius of convexity, maximum located above mid-H; PB subtriangular, sharply pointed, with narrower but still large radius of curvature, maximum located slightly below mid-H; carapace preplete; Lmax located at mid-H or slightly below.

\section{Remarks}

Orthobairdia jeanlouisi sp. nov. can be compared to Orthobairdia texana (Harlton, 1927) sensu Shi \& Chen, 2002 from the Late Permian of Heshan and Yishen, Guangxi Province, South China (Shi \& Chen 2002) but here the species is shorter, posterior maximum of convexity is clearly situated more dorsally, anterior part is more elongated, ADB steeper. Orthobairdia jeanlouisi sp. nov. is also close to Orthobairdia exilimarginata Chen, 1987 from the Changhsingian (Late Permian) of the Meishan section, Zhejiang Province, South China (Shi \& Chen 1987), but the new species is less elongated and its DB is shorter and bent backward. This species can also be related to Orthobairdia meishanensis Chen, 1987 from the Permian of the Meishan section, Zhejiang Province, South China (Shi \& Chen 1987), however, Orthobairdia jeanlouisi sp. nov. has the PB located higher, a shorter DB and a less steep PDB.

Family Pachydomellidae Berdan \& Sohn, 1961

Genus Microcheilinella Geis, 1933

Microcheilinella cf. venusta Chen, 1958

Fig. 13Q

\section{Locality}

- Wujiaping Formation (samples 05PAJ22, 24, 25, 28, 29), Dajiang section (25³3'56”N-106³9'41 '”E), Guizhou Province, South China, Changhsingian, Late Permian.

\section{Remarks}

This species is closely related to Microcheilinella venusta Chen, 1958 from the Early Permian of Lungtan, Qixia Formation, Nankin (Chen 1958) but here the specimens are more elongated and thinner, with a more rounded PB. It can also be compared to Microcheilinella cf. venusta Chen, 1958 sensu Crasquin-Soleau, 2006 from the Early Triassic of the Jinya/Waili section, Guangxi Province, South China (Crasquin-Soleau et al. 2006). However here the species is more elongated, with longer DB and no lateral compression in the posterior part. 


\author{
Superfamily Cypridacea Baird, 1845 \\ Family Paracyprididae Sars, 1923 \\ Genus Paracypris Sars, 1866
}

Paracypris gaetanii Crasquin-Soleau, 2006

Fig. 13T-X

Paracypris sp. Hao 1992: 42, pl. 1, fig. 24.

Paracypris gaetanii Crasquin-Soleau, 2006: 64, pl. 4, figs 1-4.

Paracypris sp. - Crasquin-Soleau \& Kershaw 2005: pl. 1, figs 7-9.

Paracypris gaetanii - Crasquin et al. 2008: 249, pl. 4, fig. 12. — Forel et al. 2009: 819, fig. 4 (5). — Forel \& Crasquin 2011: figs 3F', 4A.

\title{
Localities
}

- Wujiaping and Daye Formations (samples 05PAJ22, 31, 32, 35, 37-40), Dajiang section (253'56"N-106 39'41'E), Guizhou Province, South China, Changhsingian and Griesbachian, Late Permian and Early Triassic (Forel et al. 2009; this study).

- Feihsienkuan Formation, Zhenfeng, Guizhou Province, South China, Early Triassic (Hao 1992).

- Feixianguan Formation, Laolongdong section, Sichuan Province, South China, Induan, Early Triassic (Crasquin \& Kershaw 2005).

- Jinya/Waili section, Fengshan area, Guangxi Province, South China, Griesbachian, Early Triassic (Crasquin-Soleau et al. 2006).

- Bulla section, Dolomites, Southern Alps, Northern Italy, Early Triassic (Crasquin et al. 2008).

- Yinkeng Formation, Meishan section, Zhejiang Province, South China, Griesbachian, Early Triassic (Forel \& Crasquin 2011).

- Kokarkuyu Formation, Çürük dağ section, Western Taurus, Turkey, Induan, Early Triassic (Forel in progress).

\section{Remarks}

This taxon is one of the rare species which crosses the Permian - Triassic boundary.

$$
\begin{gathered}
\text { Superfamily Cytheracea Baird, } 1850 \\
\text { Family Cytherideidae Sars, } 1925 \\
\text { Genus Basslerella Kellett, } 1935
\end{gathered}
$$

Basslerella tota Chen \& Bao, 1986

Fig. 14O-Q

Basslerella tota Chen \& Bao, 1986: 123, pl. 1, figs 31-32; pl. 4, figs 7-8.

Basslerella tota - Crasquin-Soleau et al. 2004: 288, pl. 4, figs 9-10 - Yi 2004: pl. 2, fig. 20.

\section{Localities}

- Wujiaping Formation (samples 05PAJ24, 26, 28), Dajiang section (25³3'56”N-106³9'41'”E), Guizhou Province, South China, Changhsingian, Late Permian.

- Chisia formation, Jiangsu Province, South China, Early Permian (Chen \& Bao 1986).

- Pamuçak Formation, Çürük dağ section, Western Taurus, Turkey, Late Permian (Crasquin-Soleau et al. 2004).

- Kongtonshan section, Fujian Province, South China, Late Permian (Yi 2004). 

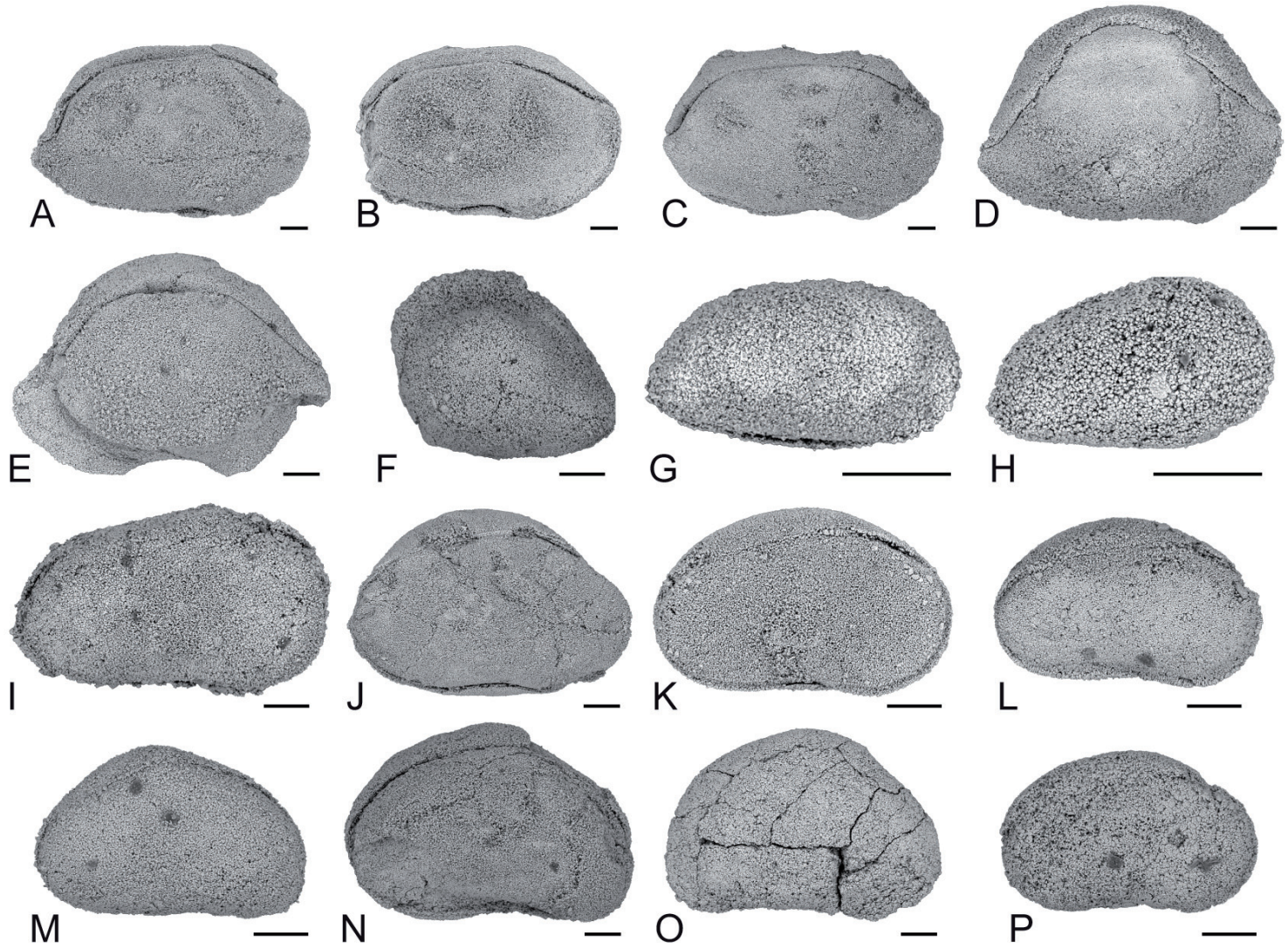

$\mathrm{L}$
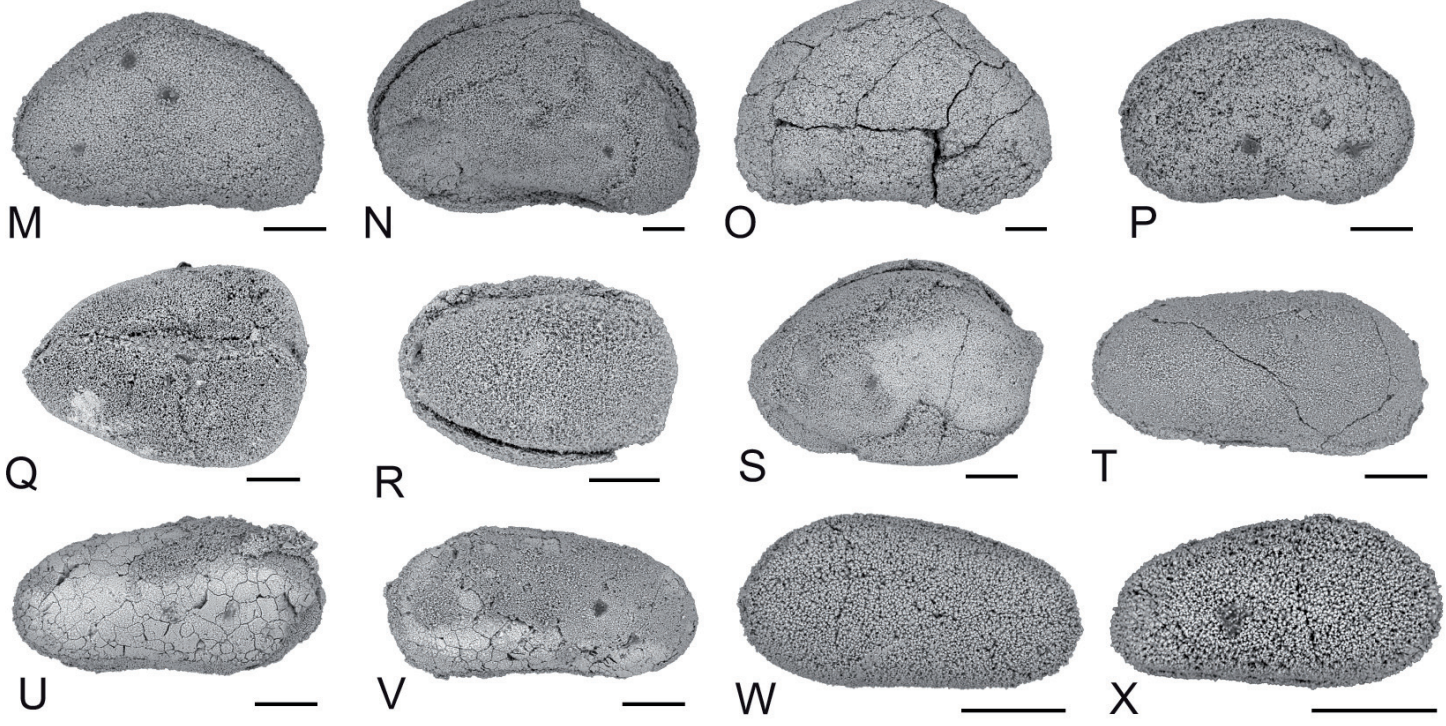

Fig. 13. Ostracods from the Dajiang section, South China. - A-B. Petasobairdia sp. 1. A. Carapace, right lateral view, P6M3082. B. Carapace, right lateral view, P6M3083. - C. Petasobairdia sp. 2, carapace, right lateral view, P6M3084. - D-E. Petasobairdia sp. 3. D. Carapace, right lateral view, P6M3085. E. Carapace, right lateral view, P6M3086. - F. Petasobairdia? sp. 4, carapace, right lateral view, P6M3087. - G. Spinocypris sp. 1, carapace, right lateral view, P6M3088. - H-I. Spinocypris? sp. 2. H. Carapace, right lateral view, P6M3089. I. Carapace, right lateral view, P6M3090. - J. Kempfina sp. 1, carapace, right lateral view, P6M3091. - K-L. Silenites sp. 1. K. Carapace, right lateral view, P6M3092. L. Carapace, right lateral view, P6M3093. - M-N. Silenites sp. 2. M. Carapace, right lateral view, P6M3094. N. carapace, right lateral view, P6M3095. - O. Silenites sp. 3, carapace, right lateral view, P6M3096. - P. Silenites sp. 4, carapace, right lateral view, P6M3097. - Q. Microcheilinella cf. venusta Chen, 1958, carapace, dorsal view, P6M3132. - R. Microcheilinella sp. 1, carapace, right lateral view, P6M3131. - S. Cetollina? sp. 1, carapace, right lateral view, P6M3098. - T-X. Paracypris gaetanii Crasquin-Soleau, 2006. T. Carapace, right lateral view, P6M3099. U. Carapace, right lateral view, P6M3100. V. Carapace, left lateral view, P6M3101. W. Carapace, left lateral view, P6M3102. X. Carapace, right lateral view, P6M3103. - Scale $=100 \mu \mathrm{m}$. 


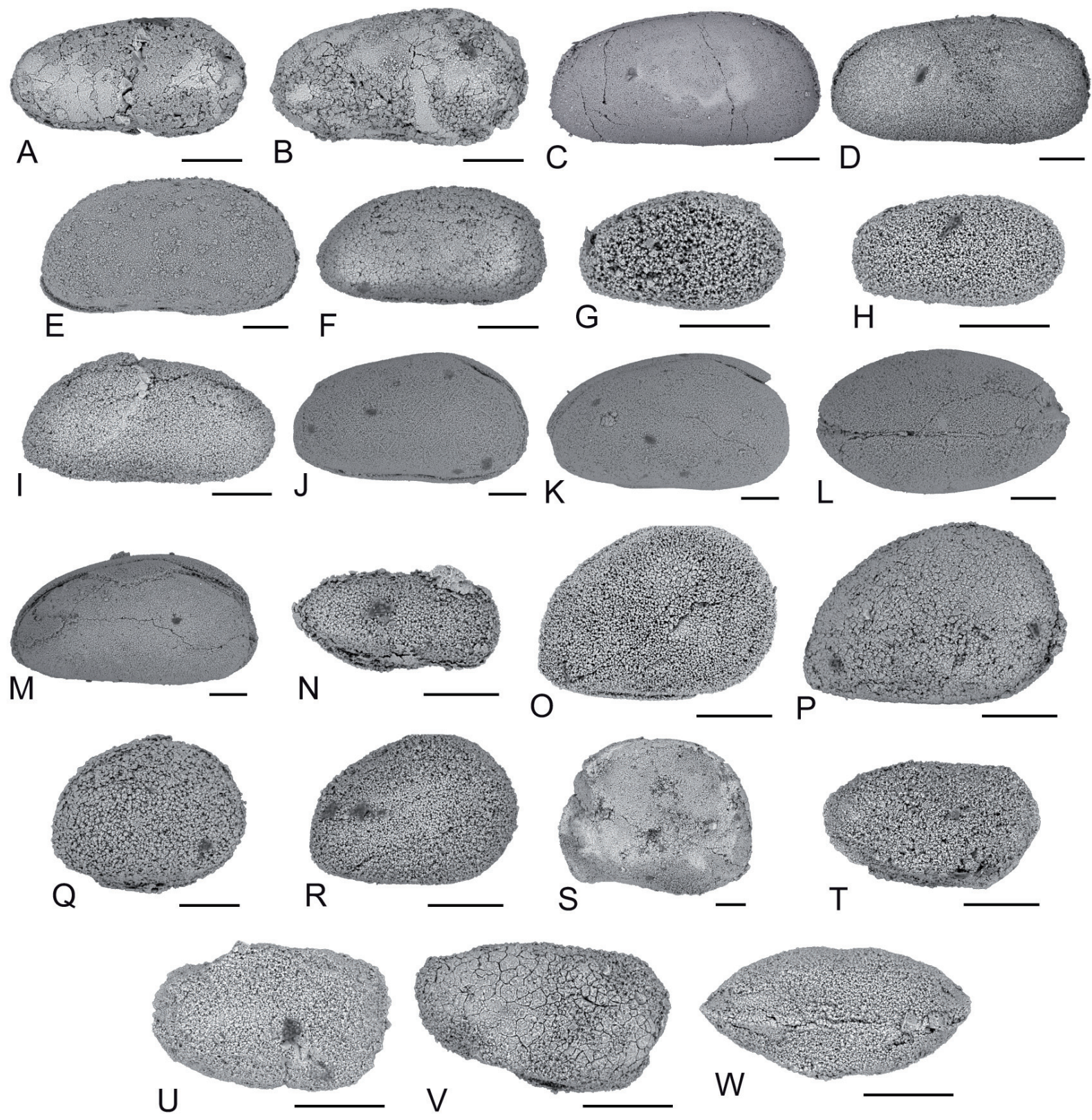

Fig. 14. Ostracods from the Dajiang section, South China. - A-B. Paracypris cf. gaetanii CrasquinSoleau, 2006. A. Carapace, right lateral view, P6M3104. B. Carapace, right lateral view, P6M3105. - C-E. Paracypris sp. 6. C. Carapace, right lateral view, P6M3106. D. Carapace, right lateral view, P6M3107. E. Carapace, right lateral view, P6M3108. - F. Paracypris sp. 1, carapace, right lateral view, P6M3109. - G. Paracypris? sp. 2, carapace, right lateral view, P6M3110. - H. Paracypris? sp. 3, carapace, right lateral view, P6M3111. - I. Paracypris? sp. 4, carapace, right lateral view, P6M3112. - J-M. Paracypris? sp. 5. J. Carapace, right lateral view, P6M3113. K. Carapace, right lateral view, P6M3114. L. Carapace, dorsal view, P6M3151. M. Carapace, right lateral view, P6M3115. - N. Monoceratina? sp. 1, carapace, right lateral view, P6M3116. - O-R. Basslerella tota Chen \& Bao, 1986. O. Carapace, right lateral view, P6M3117. P. Carapace, right lateral view, P6M3118. Q. Carapace, right lateral view, P6M3119. R. Carapace, right lateral view, P6M3120. - S. Basslerella? sp. 1, carapace, right lateral view, P6M3121. - T-W. Callicythere postiangusta Wei, 1981. T. Carapace, right lateral view, P6M3122. U. Carapace, right lateral view, P6M3123. V. Carapace, right lateral view, P6M3124. W. Carapace, dorsal view, P6M3125. - Scale $=100 \mu \mathrm{m}$. 
FOREL M.B., Ostracods from the Permian-Triassic boundary in Guizhou

Family Cytherissinellidae Kashevarova, 1958

Genus Callicythere Wei, 1981

Callicythere postiangusta Wei, 1981

Figs 14T-W, 15A-B

Callicythere postiangusta Wei, 1981: 504, pl. 1, figs 19-22.

Callicythere postiangusta - Crasquin-Soleau \& Kershaw 2005: pl. 1, figs 1-6.

\section{Localities}

- Samples 05PAJ30,31,33,35,37,38,44, Daye Formation, Dajiang section (25³3'56”N-106³9'41'”E), Guizhou Province, South China, Griesbachian, Early Triassic.

- Weiyuan, Leikoupo Formation, Sichuan Province, South China, Middle Triassic (Wei, 1981).

- Çürük dağ section, Western Taurus, Turkey, Early Triassic (Forel, in progress).

- Laolongdong section, Feixianguan Formation, Sichuan Province, South China, Early Triassic (Crasquin-Soleau \& Kershaw, 2005).

\section{Ostracod biodiversity variations}

Ostracods are found from the top of the Wujiaping formation (05PAJ20) to the base of the Daye formation (05PAJ45). Of the 26 samples within this interval, 25 yielded ostracods. Species distribution is summarised in Fig. 2 and abundance and species richness variations are presented in Fig. 16A. In the productive samples, abundance varies from 1 (05PAJ36) to 2403 (05PAJ41) and species richness from 1 (05PAJ36) to 24 (05PAJ28). A taxa-specimen rarefaction analysis has been performed and suggests that communities have been well sampled and little sampling bias is expected. Several peaks (P) and drops (D) are distinguished on the biodiversity curves (Fig. 16A):

(1) Samples 05PAJ20 to 26 are relatively highly diversified, for both species richness and abundance (P1).

(2) This phase is followed by a sharp drop from 20 species in 05PAJ25 to 2 in 05PAJ27 (D1). 05PAJ27 records the minimum of Permian ostracod diversity in Dajiang.

(3) An important diversification is observed just before the Permian - Triassic boundary (PTB) (P2: 05PAJ28, 29). This peak is the maximum of species richness in Dajiang.

(4) Samples 05PAJ28 to 05PAJ30 (D2) bracket the PTB and show an important reduction of specific richness (from 24 to 3 ).

(5) Abundance and species richness then increase from 05PAJ31 to 05PAJ33 (P3). Species richness is however lower than in the Permian assemblages.

(6) Another reduction of diversity, both abundance and specific richness, is recorded in 05PAJ34-36 (D3).

(7) A slight rediversification is observed from 05PAJ37 to 05PAJ40 (P4).

(8) A reduction of species richness, together with a high abundance, is recorded in 05PAJ41 (D4).

(9) 05PAJ42 to 05PAJ45 (P5) show a slight rediversification of the assemblages. During this phase, species richness is relatively stable while abundance is low.

The biodiversity parameters in Dajiang show striking inversion through the PTB: high species richness/ low abundance for Permian assemblages, low species richness/ high to very high abundance in Triassic microbialites. 


\section{Composition of assemblages}

Ostracods in Dajiang belong to 10 superfamilies/families, the distribution of which through the PTB is illustrated in Fig. 16B. The most abundant superfamily, both in the Permian and the Triassic, is Bairdioidea (encompassing the genera Acratia, Bairdia, Bairdiacypris, Bythocypris, Fabalicypris, Kempfina, Liuzhinia, Microcheilinella, Orthobairdia, Petasobairdia, Silenites and Spinocypris). It is absent from only 2 assemblages from D3 (05PAJ35, 36). Its proportions are between 20\% (05PAJ37) and 100\% of species (05PAJ27, 29, 45). The second most important superfamily is Cypridoidea (genus Paracypris). It is ubiquitous both in Permian and Triassic assemblages but shows higher proportions in Triassic assemblages. It is absent from 05PAJ20, 21, 26-29, 38, 44, 45. When it is present, it represents between
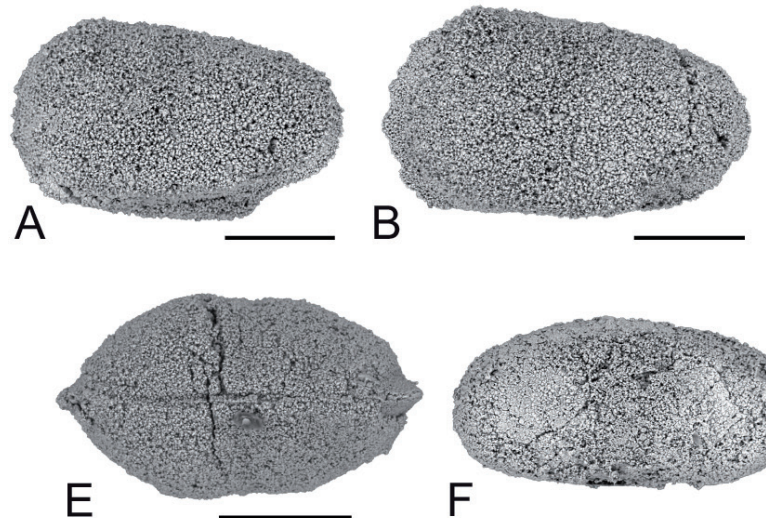

E
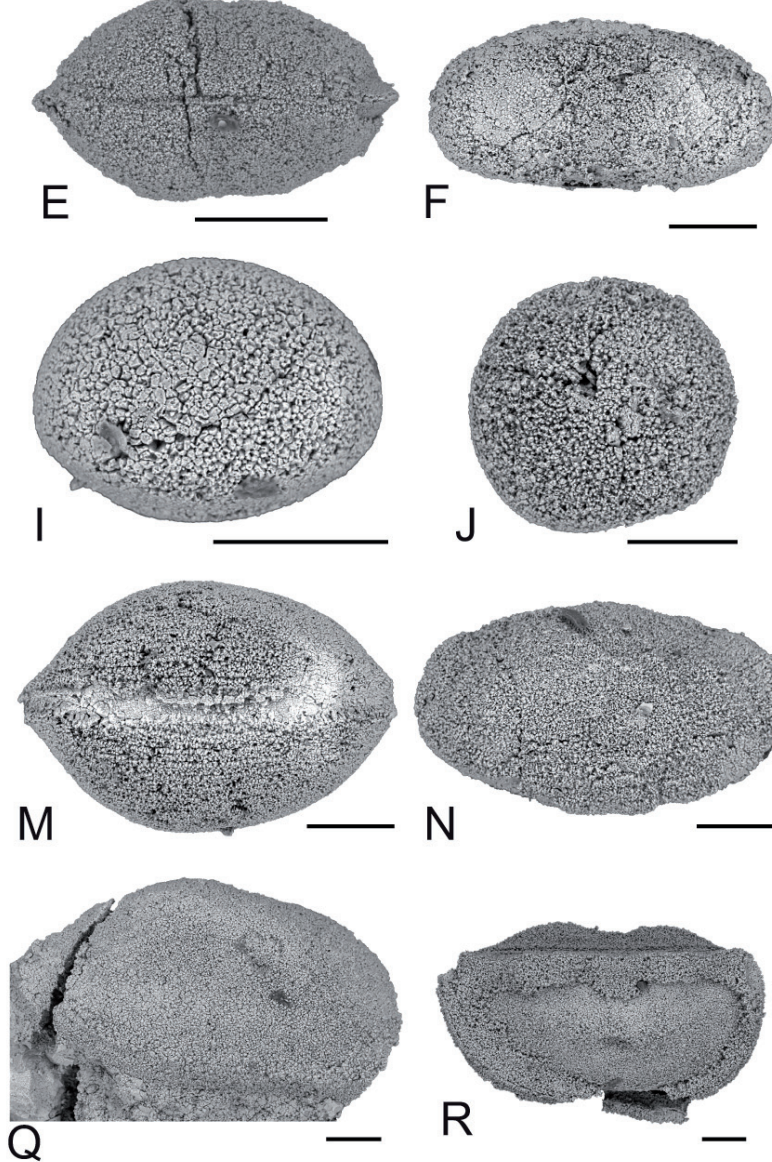

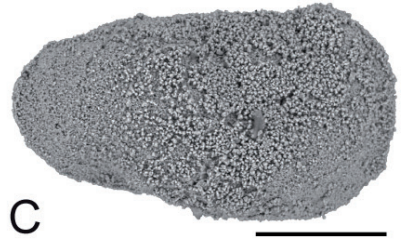

C
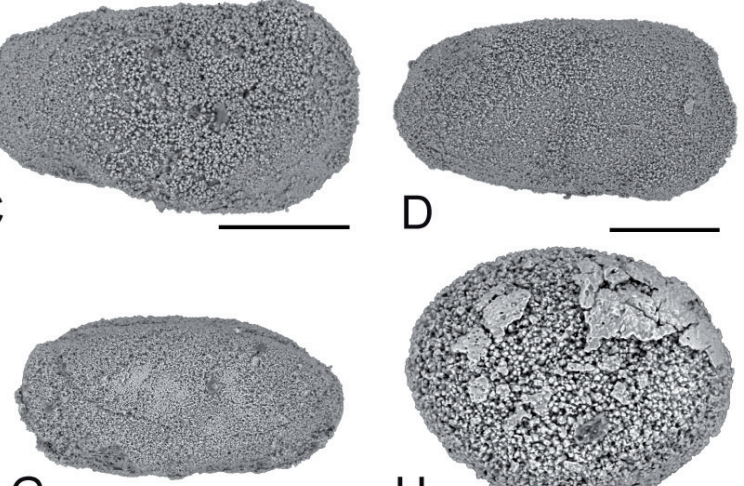

G

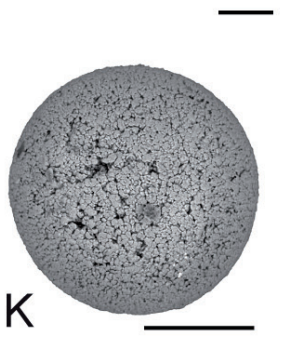

$\mathrm{H}$
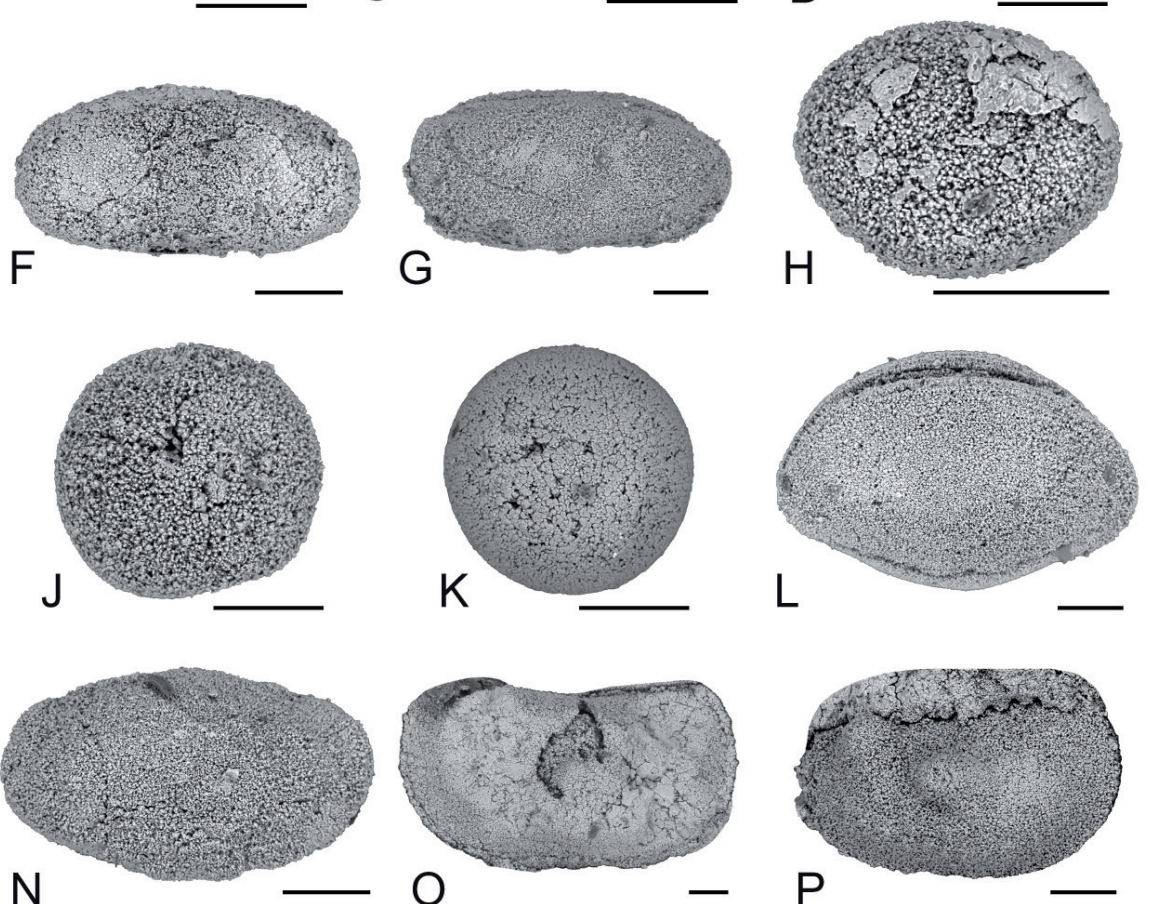

O
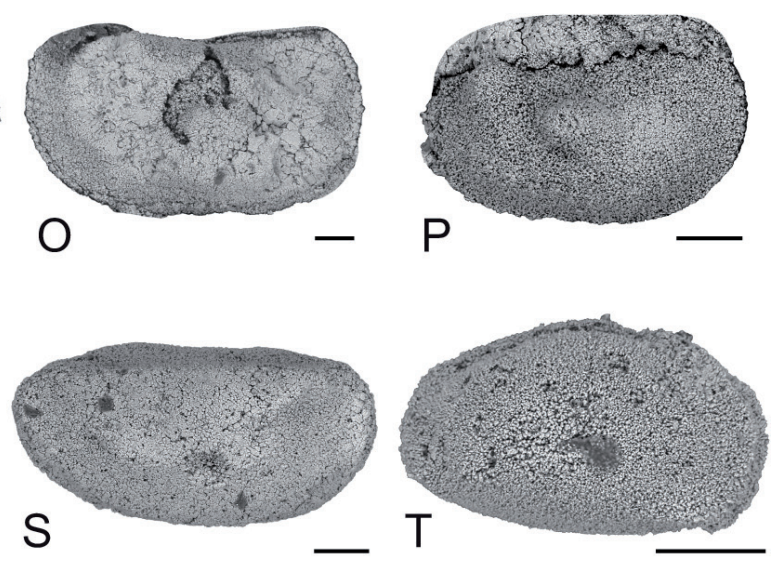
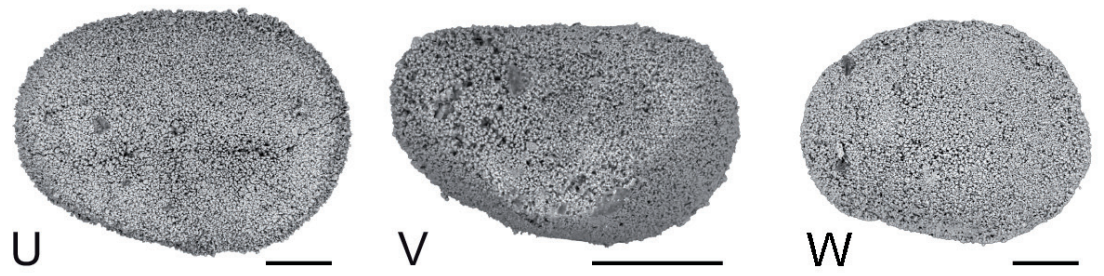
$6 \%$ (05PAJ22) and 100\% (05PAJ36) of the species. Cytheroidea (genera Basslerella, Callicythere and Monoceratina) are found both in Permian and Triassic assemblages. When it is present, it represents between 4\% (05PAJ28) and 50\% (05PAJ30, 35) of species. Paraparchitidae (genera Paraparchites, Shemonaella, Paraparchitiidae indet.) are present in 2 Permian assemblages and 1 Triassic: 05PAJ21 (11\%), 05PAJ28 (4\%), 05PAJ44 (9\%). The following superfamilies/families only occur in the Permian: Cavellinidae (genera Cavellina, Sulcella) are found in only 3 assemblages of P1 (05PAJ21, 24, 25) where they represent between 5\% (05PAJ25) and 11\% of species (05PAJ21). Kirkbyoidea (genera Amphissites, Kirkbya, Shleesha) is part of 3 assemblages from P1 (05PAJ20, 22, 24) where it is respectively 14, 6 and $20 \%$ of the species. Polycopidae (genus Polycope) is present in 2 assemblages from P1: 05PAJ24 (7\%) and 05PAJ25 (10\%). Kloedenellidea (genus Oliganisus) is found in only one assemblage (05PAJ21) where it represents $11 \%$ of the species. Aparchitidae (genus Cyathus) is present in 2 assemblages: 05PAJ21 (11\%) and 05PAJ28 (4\%). Finally, only one species belongs to an undetermined superfamily/ family (genus Cetollina). It is part of 05PAJ22 where it represents $6 \%$ of species.

In terms of species, the main extinction event occurs in the latest levels of the Wujiaping formation and the extinction rate is of $98 \%$. Two species cross the PTB in Dajiang: Bairdia jeromei sp. nov. and Paracypris gaetanii Crasquin-Soleau, 2006 (Fig. 2). They are found in almost all microbialites, their last occurrences are high in the section (respectively 05PAJ41 and 05PAJ43). Two of the ten genera found in the microbialites have not been recorded in Permian strata before: Callicythere and Orthobairdia. Consequently, the faunal turnover is nearly complete at the specific level ( $94 \%$ of new species), partial at the generic level (20\% of new genera) and no renewal is observed at the superfamily/family level. Importantly, most assemblages from the microbialites show high proportions of very small specimens (100 $\mu \mathrm{m}$ in length). These specimens belong to the same genera as the larger forms: hence the deviation of size is not due to the presence of intrinsically smaller genera. This aspect of the faunas will be addressed in future work.

\section{Discussion}

\section{Palaeoenvironmental reconstruction}

Most of the ostracods found in the Dajiang section are typical of intertropical warm settings (CrasquinSoleau et al. 1999, 2004). The presence of Polycope could indicate the influence of cold waters (Kornicker 1959). The palaeoenvironmental preferences of Late Palaeozoic and Lower Triassic ostracods (Peterson

Fig. 15. (opposite page). Ostracods from the Dajiang section, South China. - A-B. Callicythere postiangusta Wei, 1981. A. Carapace, left lateral view, P6M3126. B. Carapace, left lateral view, P6M3127. - C-E. Callicythere sp. 1. C. Carapace, right lateral view, P6M3128. D. Carapace, right lateral view, P6M3129. E. Carapace, dorsal view, P6M3130. - F. Sulcella sp. 1, carapace, right lateral view, P6M3133. - G. Sulcella? sp. 2, carapace, right lateral view, P6M3134. - H-I. Polycope sp. 1. I. Carapace, right? lateral view, P6M3135. J. Carapace, right? lateral view, P6M3136. — J. Polycope sp. 2, carapace, right? lateral view, P6M3137. - K. Polycope? sp. 3, carapace, right? lateral view, P6M3138. - L-M. Cyathus sp. 1. L. Carapace, right lateral view, P6M3139. M. Carapace, dorsal view, P6M3140. - N. Cyathus sp. 2, carapace, right lateral view, P6M3141. — O. Amphissites? sp. 1, carapace, right lateral view, P6M3142. - P. Amphissites? sp. 2, broken carapace, right lateral view, P6M3143. - Q. Shleesha? sp. 1, broken carapace, right lateral view, P6M3144. - R. Kirkbya? sp. 1, carapace, right lateral view, P6M3145. - S. Kirkbya? sp. 2, carapace, right lateral view, P6M3146. T. Oliganisus? sp. 1, carapace, left lateral view, P6M3147. - U. Paraparchites sp. 1, carapace, right lateral view, P6M3148. - V. Paraparchitidae indet., carapace, right lateral view, P6M3149. - W. Shemonaella sp. 1, carapace, left lateral view, P6M3150. - Scale $=100 \mu \mathrm{m}$. 
\& Kaesler 1980; Costanzo \& Kaesler 1987; Melnyk \& Maddocks 1988a, b; Crasquin-Soleau et al. 1999) found in Dajiang are summarized as follows (Fig. 16C):

(1) Euryhaline environments on the proximal platform (PP): Aparchitidae, Kloedenelloidea, Kirkbyoidea.

(2) Euryhaline environments, shallow to very shallow waters on the intermediate platform (IP): Cavellinidae, Cytherideidae, Paracyprididae, Paraparchitidae.

(3) Open carbonate environments with normal salinity and oygenation on the distal platform (DP): Acratiidae, Bairdiidae, Pachydomellidae.

(4) External zone of the distal platform (EDP): Bythocytheridae, Cytherissinellidae.

(5) Any platform environment, in normal salinity: Polycope.

The palaeobathymetric setting is estimated by the composition of assemblages regarding the five palaeoenvironmental groupings. Each bathymetrical zone is marked by the relative dominance of the corresponding group, while relative abundances of the other groups give information about the positioning within the zone determined by the dominant group.

DP forms are found all along the section and are always more than $50 \%$ of the assemblages (Fig. 16C). They indicate an open marine environment both in Late Permian and Early Triassic microbialites in Dajiang. PP forms only occur in Permian assemblages (05PAJ20-22, 24, 28). IP forms show the same pattern (05PAJ21, 24-26, 28) with one Triassic occurrence (05PAJ44). This documents proximal and variable conditions in the Permian of Dajiang, in the internal part of the circalittoral stage. EDP forms appear significantly in the first Triassic assemblage and are found throughout the microbialites. This suggests that the environmental stabilisation through the PTB can be related to a transgressive trend. Polycope are found only in Permian assemblages together with the unique Permian occurrence of EDP forms. This co-existence records a marked deepening of the area, associated with the possible influence of cold waters. The quasi-absence of PP and IP forms in the microbialites indicates a relative environmental stability in the deep external circalittoral zone, as revealed by the overwhelming dominance of DP forms and with significant components of EDP forms.

Worthy to note is the co-occurrence in assemblages 05PAJ25 and 44 of IP and EDP forms, indicating very different environmental settings. This record is best explained by considering that ecological classes used in this study not only reflect bathymetry but more generally the stability of the environmental parameters, here salinity, oxygenation and water depth. The punctual co-occurrence of these IP and EDP therefore seems to be related to the decoupling of environmental factors.

In summary, ostracod data provide additional evidence for the open-marine context through the entire PTB interval in Dajiang (Lehrmann et al. 2005). The shallow-subtidal, open-marine platform environment with relatively low to moderately high current energy of the Wujiaping formation is documented by ostracod faunas. However, the transgressive trend described here is discordant compared to the sedimentological proxy that indicates environments similar to the underlying Permian skeletal packstone. This discrepancy can be related to the fact that wave action has been reconstructed based on interbedded molluscan grainstone, which may only be episodic phenomenon (Lehrmann et al. 2005).

\section{Contribution to the debate on oxygenation}

As stated above, oxygenation of marine waters surrounding microbialites is an important challenge to understand their build-up mechanisms and the survival/recovery phenomenon following the extinction. In our former work addressing ostracod faunas of the PTB transition in Dajiang, we used the traditional Lethiers \& Whatley model (Lethiers \& Whatley 1994) to estimate the oxygen concentration at the base of the water column (Forel et al. 2009). This tool is now highly questioned and it is no longer possible to use it (e.g. Brandão \& Horne 2009), but we can get a qualitative idea of the water oxygenation 
independently. Most Permian and Triassic assemblages from Dajiang are dominated by Bairdioidea (being more than $50 \%$ of species, except in $05 \mathrm{PAJ} 30,35-37,40,41$; Forel in press). This superfamily has been associated throughout its history with normal oxygenation settings ( $\mathrm{Pr}$ R. Maddocks, Univ. of Houston, pers. comm.), so its dominance is a witness of good oxygenation at the base of the water
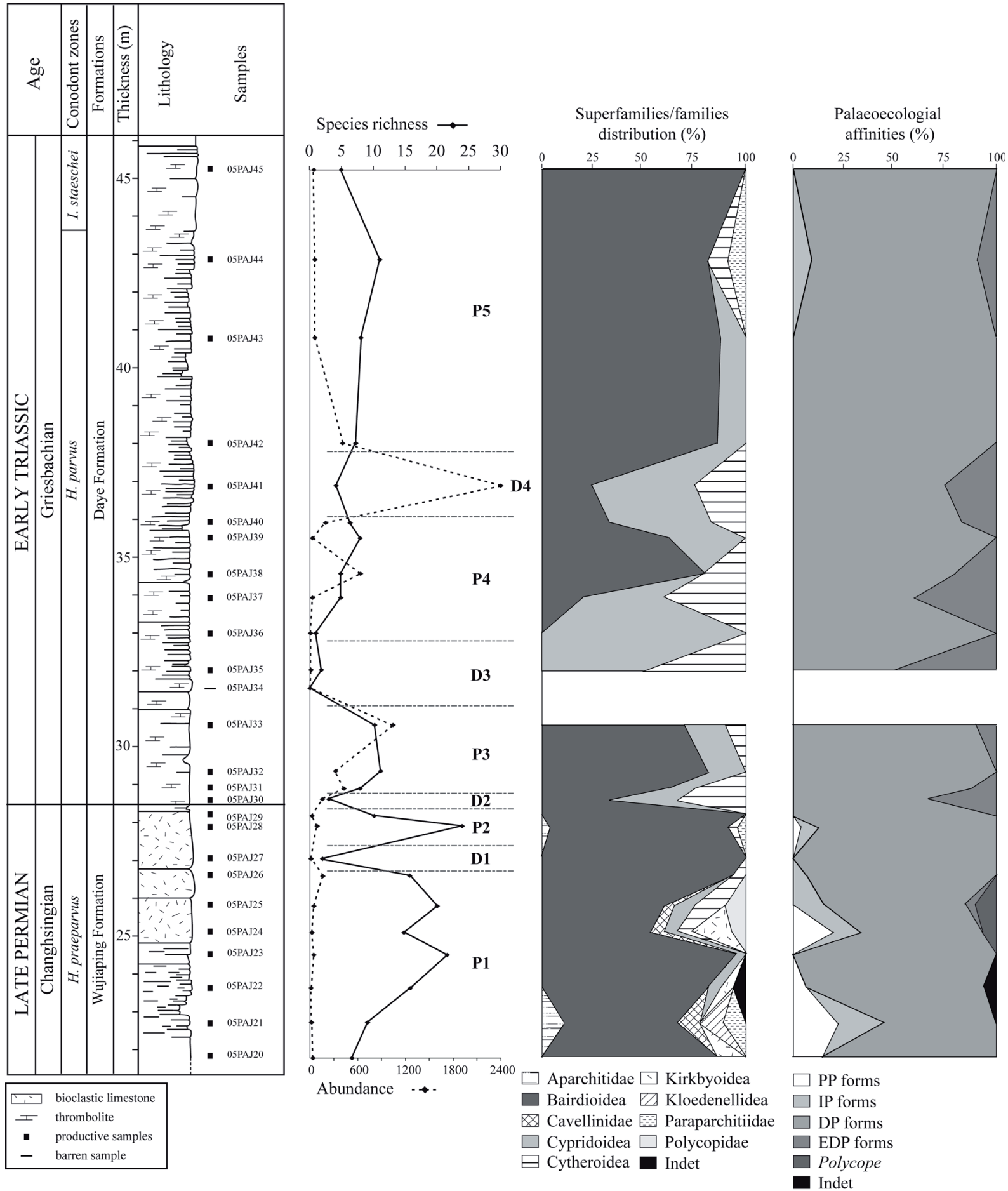

Fig. 16. Evolution of the ostracod faunas through the PTB in Dajiang. A. Evolution of the number of species (species richness) and number of specimens (abundance). B. Evolution of the relative proportions of each superfamily/family. C. Evolution of the relative proportions of each palaeoecological group. 
column. The assemblages where Bairdioidea no longer represent the majority yielded high proportions of Cypridoidea that have similar requirements regarding oxygen levels (Pr R. Maddocks, Univ. of Houston pers. comm.). Although the setting through the PTB in Dajiang appears as normoxic, the biodiversity changes described above undeniably indicate deep ecological modifications. They should be linked to the relative degradation of the environment, buffered by the refuge created by the microbial ecosystem (Forel in press). These peculiar assemblages are recognised in all microbialites bearing sections analysed until now while they are absent from synchronous non-microbial deposits from deeper settings such as at the Meishan section, Global Stratotype Section and Point of the PTB (Crasquin et al. 2010; Forel \& Crasquin 2011). They are intimately linked to the presence of microbialites $(i)$ in space, areas lacking microbial mats are devoid of ostracods, (ii) in time, faunas disappearing with microbialites (e.g. Forel et al. in press; Forel in press).

\section{Note on palaeo-geographical distributions and marine ostracods dispersal ways}

Several species of this study display a wide distribution around both the Palaeo-Tethys and Neo-Tethys Oceans: Bairdiacypris Crasquin-Soleau, 2004, Liuzhinia antalyaensis Crasquin-Soleau, 2004, Paracypris gaetanii Crasquin-Soleau, 2006, Basslerella tota Chen \& Bao, 1986, Callicythere postiangusta Wei, 1981. Because benthic ostracods have no pelagic stage, their migration is performed actively through locomotion and/or passively by the bottom currents. Their carbonate carapaces exclude them from depths below the lysocline, their migration is achieved on routes above this level. Their dispersal relies on the constancy of favourable environmental parameters of water-masses on their route, e.g. oxygen content, salinity, temperature.

However, active transoceanic dispersal potential of recent forms is relatively limited. Eggs, juveniles and adults would therefore be passively carried by wind, birds, fish, drifting algae or oceanic currents (Van Morkhoven 1962; Sandberg 1964; Teeter 1973; Whatley 1988; Babinot \& Colin 1992; Lethiers \& Crasquin-Soleau 1995). The characteristics of eggs of marine ostracods set some limits to this dissemination mode. Whereas eggs of freshwater ostracods are double-walled, those of marine ostracods are single-walled and do not stand desiccation (Kesling 1961), excluding dispersal by birds and winds (Teeter 1973). Larvae and adults are less sensitive to wind transportation because of their bigger size but would probably not withstand it (Teeter 1973). Although Kornicker \& Sohn (1971) showed the possibility for adult freshwater ostracods ingested by fish to survive, it is unlikely that marine forms would have the same resistance. Aquatic plants could also be an important transportation vector for ostracods. Teeter (1973) found living specimens of Hemicytherura cranekeyensis Puri, 1960 on the marine algae Turbinaria Lamouroux, 1825 from Honduras. These algae often break into pieces that can float very far from the coast. Living ostracods have also been observed on the algae Sargassum Agardh, 1820. Turbinaria and Sargassum are found in the tropical area of Atlantic and Pacific Oceans. Once floating they could drift under the influence of surface currents to climatic zones favorable or not to the survival of the commensal ostracods. Most of the living ostracods have a high degree of flexibility and ecological tolerance so they should have a high capacity to successfully invade new environments.

\section{Acknowledgements}

This study is part of IGCP 572 'Restoration of Marine Ecosystems following the Permian-Triassic Mass Extinction: lessons for the present' and was undertaken with the support of the French CNRS Research Team UMR 7207 CR2P and the Chinese programs NSFC (40839903 and 40921062) and 111 (B08030). I am deeply indebted to my PhD supervisor Dr. Sylvie Crasquin (CNRS - CR2P) for her availability and great help in this study. I thank Prof. Feng Qinglai (China University of Geosciences, Wuhan) for his help during fieldwork on the Dajiang section. I also thank Martine Fordant (UPMC, Paris) for the processing of material and Alexandre Lethiers (UPMC, Paris) for his help with the illustrations. I am also grateful to Dr. Carys Bennett (Universite de Lille, France) and Dr. Vincent Perrier (University of 
Tartu, Estonia) for their critical reviews and constructive suggestions, which have greatly improved the quality of this analysis.

\section{References}

Babinot J.F. \& Colin J.P. 1992. Marine ostracode provincialism in the Late Cretaceous of the Tethyan realm and the Austral Province. In: Malmgren B.A. \& Bengtson P. (eds) Biogeographic patterns in the Cretaceous ocean. 5th biannual meeting of the European Union of Geosciences Symposium on Biogeographic patterns in the Cretaceous ocean: 283-293. Strasbourg, France.

Benton M.J. \& Twitchett R.J. 2003. How to kill (almost) all life: the end-Permian extinction event. Trends in Ecology \& Evolution 18: 358-365.

Bond D.P.G., Wignall P.B., 2010. Pyrite framboid study of marine Permo-Triassic boundary sections: a complex anoxic event and its relationship to contemporaneous mass extinction. Geological Society of America Bulletin 122: 1265-1279. http://dx.doi.org/ 10.1130/B30042.1

Brandão S.N. \& Horne D.J. 2009. The Platycopid Signal of oxygen depletion in the ocean: a critical evaluation of the evidence from modern ostracod biology, ecology and depth distribution. Palaeogeography, Palaeoclimatology, Palaeoecology 283: 126-133. http://dx.doi.org/10.1016/j. palaeo.2009.09.007

Chen D.Q. \& Bao H. 1986. Lower Permian Ostracodes from the Chihsia Formation of Jurongan Longtar, Jiangsu Province. Acta Micropalaeontologica Sinica 3: 107-132.

Chen L., Wang Y., Xie S., Kershaw S., Dong M., Yang H., Liu H., Algeo T.J. 2011. Molecular records of microbialites following the end-Permian mass extinction in Chongyang, Hubei Province, South China. Palaeogeography, Palaeoclimatology, Palaeoecology 308: 151-159. http://dx.doi.org/10.1016/j. palaeo.2010.09.010

Chen T.C. 1958. Permian ostracods from the Chihsia limestone of Lungtan, Nanking. Acta Palaeontologica Sinica 6: 215-257.

Costanzo G.V. \& Kaesler R.L. 1987. Changes in Permian marine Ostracode faunas during regression, Florena Shale, Northeastern Kansas. Journal of Paleontology 61: 1204-1215.

Crasquin S., Forel M.B., Feng Q., Yuan A., Baudin F. \& Collin P.Y. 2010. Ostracods (Crustacea) through the Permian-Triassic boundary in South China: the Meishan stratotype (Zhejiang Province). Journal of Systematic Palaeontology 8: 331-370. http://dx.doi.org/10.1080/14772011003784992

Crasquin S., Perri M.C., Nicora A. \& De Wever P. 2008. Ostracods across the Permian - Triassic boundary in Western Tethys: the Bulla parastratotype (Southern Alps). Rivista Italiana di paleontologia e Stratigrafia 114: 235-264.

Crasquin-Soleau S., Broutin J., Roger J., Platel J.-P., Al Hashmi A., Angiolini L., Baud A., Bucher H. \& Marcoux J. 1999. First Permian ostracode fauna from the Arabian Plate (Khuff Formation, Sultanate of Oman). Micropaleontology 45: 163-182.

Crasquin-Soleau S., Galfetti T. \& Bucher H. 2006. Early Triassic ostracods from South China. Rivista Italiana di Paleontologia e Stratigrafia 112: 55-75.

Crasquin-Soleau S. \& Kershaw S. 2005. Ostracod fauna from the Permian-Triassic boundary interval of South China (Huaying Mountains, eastern Sichuan Province): palaeoenvironmental significance. Palaeogeography, Palaeoclimatology, Palaeoecology 217: 131-141. http://dx.doi.org/10.1016/j. palaeo.2004.11.027 
Crasquin-Soleau S., Marcoux J., Angiolini L., Richoz S., Nicora A., Baud A. \& Bertho Y. 2004. A new ostracode fauna from the Permian-Triassic boundary in Turkey (Taurus, Antalya Nappes). Micropaleontology 50: 281-296.

Crasquin-Soleau S., Vaslet D. \& Le Nindre Y.M. 2005. Ostracods as markers of the Permian/Triassic boundary in Khuff Formation of Saudi Arabia. Palaeontology 48: 853-868. http://dx.doi.org/10.1111/ j.1475-4983.2005.00476.x

Erwin D.H. 1993. The Great Paleozoic Crisis: Life and Death in the Permian. Columbia University Press, New York.

Forel M.B. in press. The Permian-Triassic mass extinction: ostracods (Crustacea) and microbialites. Comptes Rendus Geoscience.

Forel M.B. \& Crasquin S. 2011. Lower Triassic ostracods (Crustacea) from Meishan section, Permian - Triassic GSSP (Zhejiang Province, South China). Journal of Systematic Palaeontology 9: 455-466. http://dx.doi.org/10.1080/14772019.2010.526638

Forel M.B., Crasquin S., Hips K., Kershaw S., Collin P.Y., Haas J. in press. Ostracods (Crustacea) from the Permian-Triassic Boundary of Bükk Mountains (Hungary). Acta Paleontologica Polonica.

Forel M.B., Crasquin S., Kershaw S., Feng Q.L. \& Collin P.Y. 2009. Ostracods (Crustacea) and water oxygenation in earliest Triassic of South China: implications for oceanic events of the endPermian mass extinction. Australian Journal of Earth Sciences 56: 815-823. http://dx.doi.org/ $10.1080 / 08120090903002631$

Hao W.C. 1992. Lower Triassic marine ostracods from Guizhou. Acta Micropaleontologica Sinica 9: $37-44$.

Kershaw S., Crasquin S., Li Y., Collin P.Y., Forel M.B., Mu X., Baud A., Wang Y., Xie S., Guo L., Maurer F. 2012. Microbialites and global environmental change across Permian - Triassic boundary: a synthesis. Geobiology 10 (1): 25-47. http://dx.doi.org/10.1111/j.1472-4669.2011.00302.x

Kershaw S., Li, Y., Crasquin-Soleau S., Feng Q., Mu X., Collin P.Y., Reynolds A., Guo L., 2007. Earliest Triassic microbialites in the South China block and other areas: control on their growth and distribution. Facies 53: 409-425. http://dx.doi.org/10.1007/s10347-007-0105-5

Kesling R.V. 1961. Morphology of living Ostracodes. In: Moore R.C. (ed.) Arthropoda 3. Crustacea, Ostracoda: Q3-Q17. Geological Society of America and University of Kansas Press; Boulder, Lawrence.

Kornicker L.S. 1959. Distribution of the ostracode suborder Cladocopa and a new species from the Bahamas. Micropaleontology 5: 69-75.

Kornicker L.S. \& Sohn I.G. 1971. Viability of Ostracode Eggs Egested by Fish and Effect of Digestive Fluids on Ostracode Shells - Ecologic and Paleoecologic Implications. Bulletin Centre Recherche Pau - SNPA 5: 125-135.

Kozur H. 1985. Neue ostracoden-Arten aus dem oberen Mittelkarbon (Höheres Moskovian) Mittel- und Oberperm des Bükk-Gebirges (N-Ungarn). Geologisch-Paläontologische Mitteilungen 2: 1-145.

Lehrmann D.J. 1999. Early Triassic calcimicrobial mounds and biostromes of the Nanpanjiang basin, South China. Geology 27 (4): 359-362. http://dx.doi.org/10.1130\%2F0091-7613\%281999\%29027\%3C0 359\%3AETCMAB $\% 3 \mathrm{E} 2.3 . \mathrm{CO} \% 3 \mathrm{~B} 2$

Lehrmann D.J., Payne J.L., Enos P., Montgomery P., Wei J., Yu Y., Xiao J. \& Orchard M.J. 2005. Field excursion 2: Permian-Triassic boundary and a Lower-Middle Triassic boundary sequence on the Great Bank of Guizhou, Nanpanjiang basin, southern Guizhou Province. Albertiana 33: 169-186. 
Lehrmann D.J., Payne J.L., Felix S.V., Dillett P.M., Wang H., Yu Y. \& Wei J. 2003. Permian-Triassic boundary sections from shallow-marine carbonate platforms of the Nanpanjiang basin, south China: implications for oceanic conditions associated with the end-Permian extinction and its aftermath. Palaios 18: 138-152. http://dx.doi.org/10.1669\%2F0883-1351\%282003\%2918\%3C138\%3APBSFSC \%3E2.0.CO\%3B2

Lethiers F. \& Crasquin-Soleau S. 1988. Comment extraire des microfossiles à tests calcitiques de roches calcaires dures. Revue de Micropaléontologie 31: 56-61.

Lethiers F. \& Crasquin-Soleau S. 1995. Distribution des ostracodes et paléocourantologie au Carbonifère terminal-Permien. Geobios 17: 257-272.

Lethiers F. \& Whatley R. 1994. The use of Ostracoda to reconstruct the oxygen levels of the Late Paleozoic oceans. Marine Micropaleontology 24: 57-69.

Liao W., Wang Y., Kershaw S., Weng Z., Yang H. 2010. Shallow-marine dysoxia across the PermianTriassic boundary: evidence from pyrite framboids in the microbialite in South China. Sedimentary Geology 232: 77-83.

Melnyk D.H. \& Maddocks R.F. 1988a. Ostracode biostratigraphy of the Permo-Carboniferous of central and north-central Texas, Part I: paleoenvironmental framework. Micropaleontology 34: 1-20.

Melnyk D.H. \& Maddocks R.F. 1988b. Ostracode biostratigraphy of the Permo-Carboniferous of central and north-central Texas, Part II: ostracode zonation. Micropaleontology 34: 21-40.

Mette W. 2008. Upper Permian and lowermost Triassic stratigraphy, facies and ostracods in NW Iran implications for the P/T extinction event. Stratigraphy 5: 205-219.

Payne J.L. \& Clapham M.E. 2012. End-Permian Mass Extinction in the Oceans: An Ancient Analog for the Twenty-First Century? Annual Review of Earth and Planetary Sciences 40: 89-111.

Peterson R.M. \& Kaesler R.L. 1980. Distribution and diversity of ostracodes assemblages from the Hamlin Shale and the Americus limestones (Permian, Wolfcampian) in northern Kansas. University of Kansas Paleontological Contributions 100: 1-26.

Sandberg P.A. 1964. The Ostracod genus Cyprideis in the Americas. Stockholm Contributions in Geology 7.

Sepkoski J.J.J. 1984. A kinetic model of Phanerozoic taxonomic diversity; III, Post Paleozoic families and mass extinctions. Paleobiology 10 (2): 246-267.

Shi C.G. \& Chen D.Q. 1987. The Changhsingian ostracodes from Meishan, Changxing, Zhejiang. Stratigraphy and Palaeontology of Systemic Boundaries in China; Permian and Triassic Boundary 5: 23-80 [in Chinese with English abstract].

Shi C.G. \& Chen D.Q. 2002. Late Permian ostracodes from Heshan and Yishan of Guangxi. Bulletin of the Nanjing Institute of Geology and Paleontology 15: 47-129 [in Chinese with English abstract].

Song H.J., Tong J., Chen Z.Q., Yang H. \& Wang Y.B. 2009. End-Permian mass extinction of foraminifers in the Nanpanjiang Basin, South China. Journal of Paleontology 83: 718-738.

Teeter J.W. 1973. Geographic distribution and dispersal of some recent shallow water marine Ostracoda. Ohio Journal of Science 73 (1): 46-54.

Van Morkhoven F.P. 1962. Post Paleozoic Ostracoda. Their morphology, taxonomy and economic use. 1- General. Elsevier, Amsterdam.

Wang S. 1978. Late Permian and Early Triassic ostracods of Western Guizhou and Northeastern Yunnan. Acta Palaeontologica Sinica 17: 277-308. 
Wei M. 1981. Early and Middle Triassic Ostracods from Sichuan. Acta Palaeontologica sinica, 20 (6): 501-510 [in Chinese with English abstract].

Whatley R.C. 1988. Ostracoda and paleogeography. In: De Deckker P., Colin J.P. \& Peypouquet J.P. (eds) Ostracoda in Earth Sciences: 103-123. Elsevier, Amsterdam.

Yang H., Chen Z.Q., Wang Y., Tong J., Song H., Chen J. 2011. Composition and structure of microbialite ecosystems following the end-Permian mass extinction in South China. Palaeogeography, Palaeoclimatology, Palaeoecology 308: 111-128. http://dx.doi.org/10.1016/j.palaeo.2010.05.029

Yi W. 2004. Ostracodes from the Upper Permian and Lower Triassic at the Kongtongshan scetion of Datian, Fujian. Acta Palaeontologica Sinica 43: 556-570.

Yin H., Wu S., Ding M., Zhang K., Tong J., Yang F. \& Lai X. 1996. The Meishan section, candidate of the Global Stratotype Section and Point of Permian-Triassic boundary. In: Yin H.F (ed.) The PaleozoicMesozoic Boundary Candidates of the Global Stratotype Section and Point of the Permian-Triassic Boundary: 31-48. China University of Geosciences Press, Wuhan.

Manuscript received: 3 April 2012

Manuscript accepted: 26 June 2012

Published on: 1 August 2012

Topic editor: Christian de Muizon

In compliance with the $I C Z N$, printed versions of all papers are deposited in the libraries of the institutes that are members of the EJT consortium: Muséum National d'Histoire Naturelle, Paris, France; National Botanic Garden of Belgium, Meise, Belgium; Royal Museum for Central Africa, Tervuren, Belgium; Natural History Museum, London, United Kingdom; Royal Belgian Institute of Natural Sciences, Brussels, Belgium; Natural History Museum of Denmark, Copenhagen, Denmark. 